\title{
An improved theory for calculating leaf gas exchange more precisely accounting for small fluxes
}

\author{
Diego A. Márquez $\odot$, Hilary Stuart-Williams $\odot$ and Graham D. Farquhar $\odot \bowtie$
}

The widely used theory for gas exchange proposed by von Caemmerer and Farquhar (vCF) integrates molar fluxes, mole fraction gradients and ternary effects but does not account for cuticular fluxes, for separation of the leaf surface conditions or for ternary effects within the boundary layer. The magnitude of cuticular conductance to water $\left(g_{\mathrm{cw}}\right)$ is a key factor for determining plant survival in drought but is difficult to measure and often neglected in routine gas exchange studies. The vCF ternary effect is applied to the total flux without the recognition of different pathways that are affected by it. These simplifications lead to errors in estimations of stomatal conductance, intercellular carbon dioxide concentration $\left(C_{i}\right)$ and other gas exchange parameters. The theory presented here is a more precise physical approach to the electrical resistance analogy for gas exchange, resulting in a more accurate calculation of gas exchange parameters. Additionally, we extend our theory, using physiological concepts, to create a model that allows us to calculate cuticular conductance to water.

G as exchange between a leaf and the atmosphere occurs simultaneously through two pathways: (1) epidermal cells and cuticular waxes (henceforth referred to as 'cuticle') which are always present at each leaf surface and (2) stomata, which typically control most of the exchange. The gradients that drive the gas exchange are the differences between concentrations inside the leaf and the surfaces of the leaf, and between the leaf surfaces and the external air. The ternary effect applies to the fluxes between the surfaces of the leaf and the external air and to the fluxes crossing the stomata but not to the fluxes crossing the cuticle. However, in the von Caemmerer and Farquhar ${ }^{1}$ theory (vCF), the gas exchange is considered as fluxes passing through the stomata and the boundary layer with direct connection between the inside and the external environment, and ternary corrections are applied to the whole flux."

In calculations of gas exchange parameters, measurements of transpiration rate are used to estimate conductances to water and those estimations are widely used to calculate conductances to $\mathrm{CO}_{2}$ $\left(\right.$ ref. $\left.^{2}\right)$. Thus, to differentiate the fluxes from the leaf surfaces, it is necessary to define the cuticular conductance to $\mathrm{H}_{2} \mathrm{O}\left(g_{\mathrm{cw}}\right)$ and stomatal conductance to $\mathrm{H}_{2} \mathrm{O}\left(g_{\mathrm{sw}}\right)$. However, it is typically impracticable to identify $g_{\mathrm{cw}}$ and $g_{\mathrm{sw}}$ separately when the flux of water occurs through both at the same time ${ }^{3}$. Then, as stomata dominate gas exchange, $g_{\mathrm{cw}}$ is for convenience commonly treated as being negligible ${ }^{4}$.

Overlooking $g_{c w}$ is problematic because, in some cases, it can be as high as $28 \%$ of the maximum stomatal conductance ${ }^{5}$ or the stomatal conductance to $\mathrm{H}_{2} \mathrm{O}$ vapour $\left(g_{\text {sw }}\right)$ can be low enough to magnify the importance of $g_{\mathrm{cw}}\left(\right.$ ref. $\left.^{6}\right)$. Additionally, cuticular transpiration is important under dry conditions when it contributes to desiccation and death of leaves whose stomata are already $\operatorname{closed}^{7,8}$. In practice, the assumption that cuticular conductance is negligible leads to the miscalculation of stomatal water fluxes and $\mathrm{CO}_{2}$ fluxes deduced from them, including the internal $\mathrm{CO}_{2}$ concentration $\left(C_{\mathrm{i}}\right)$ and all the subsequent calculations that involve these parameters ${ }^{9}$ (for example, mesophyll conductance ${ }^{10}$ ). Thus, cuticular conductance to water is a key unknown when calculating gas exchange parameters more precisely ${ }^{9,11,12}$.
Cuticular conductance is often estimated from measurements of leaf conductance when stomata are tending to close, such as in darkness ${ }^{13}$, obtaining the 'minimum' leaf conductance. The 'minimum' leaf conductance is, however, the sum of the cuticular conductance and additions from stomata which may be incompletely closed ${ }^{13}$. This situation can be improved by adding abscisic acid (ABA) to promote stomatal closure, so that gas exchange becomes dominated by the cuticle ${ }^{11}$. An additional measurement difficulty is that cuticular conductance changes with leaf water status ${ }^{6,14}$. Boyer ${ }^{14}$ observed that when the leaf dries out, epidermal cells shrink, affecting the cuticular conductance to $\mathrm{H}_{2} \mathrm{O}$ and $\mathrm{CO}_{2}$. This highlights the importance of obtaining values of cuticular conductance under the same conditions as those of the experiment being conducted or, better, during the measurements themselves.

Boyer ${ }^{11}$ and Tominaga et al. ${ }^{15}$ presented a technique to estimate cuticular conductance to water from the difference between two estimations of conductance to $\mathrm{CO}_{2}$. One from direct measurements of $C_{\mathrm{i}}$ from the abaxial surface $\left(g_{\mathrm{tc}-\mathrm{m}}\right)$ and a second using vCF cal- $\mathrm{Q} \mathrm{z}$ culations of total conductance to $\mathrm{CO}_{2}$ through the adaxial surface $\left(g_{\mathrm{tc}-\mathrm{vCF}}\right)\left(\right.$ ref. $\left.{ }^{12}\right)$ as $g_{\mathrm{cw}}=1.6 \times\left(g_{\mathrm{tc}-\mathrm{vCF}}-g_{\mathrm{tc}-\mathrm{m}}\right)$, on the basis of the tacit assumption of adaxial and abaxial $C_{\mathrm{i}}$ being equal. Equations used by Tominaga ${ }^{12}$ and Boyer ${ }^{11}$ differ slightly but both are based on the von Caemmerer and Farquhar ${ }^{1}$ (vCF) theory. This is a convenient simplification to obtain a $g_{\mathrm{cw}}$ value where it is assumed that the difference between the calculated and measured $g_{\mathrm{tc}}$ is only due to the vCF theory neglecting cuticular conductance to water. However, the vCF theory does not include any variable describing $\mathrm{H}_{2} \mathrm{O}$ and $\mathrm{CO}_{2}$ fluxes through the cuticle, nor the interaction between cuticular fluxes and stomatal and boundary layer fluxes.

In our study, a theory of gas exchange is developed including cuticular fluxes and their interaction with the other gas exchange parameters, the conditions at the leaf surface and ternary corrections in the boundary layer. To evaluate the theory proposed here, a model to calculate cuticular conductance to water was derived from the theory and a set of experiments was performed to obtain $g_{\mathrm{cw}}$ using our model. These experiments were largely performed on a 
system created by S.C. Wong ${ }^{16,17}$ that measures adaxial and abaxial gas exchange independently (system 1). Further tests were made using an inhouse setup of two LI-6800s that emulate system 1 dual measurements.

Here, we examine the basis of usual leaf gas exchange calculations, then introduce the main theory proposed for calculation of gas exchange parameters. Finally, we extend the theory by adding physiological assumptions to create a model that allows us to calculate cuticular conductance. The technique and the assumptions made are then examined.

A list of the abbreviations and subscripts used in this paper is shown in Supplementary Note 1.

\section{The basis of current theory of leaf gas exchange}

The total conductance to $\mathrm{H}_{2} \mathrm{O}\left(g_{\mathrm{tw}}\right)$ is conventionally estimated from the evaporative flux ${ }^{2,18}\left(E_{\mathrm{T}}\right)$ by using equations introduced by von Caemmerer and Farquhar ${ }^{1}$ (vCF). The vCF theory was the first to combine the use of molar fluxes, mole fraction gradients and ternary corrections (including the effect of the evaporative flux on itself and on $\mathrm{CO}_{2}$ fluxes) but is silent regarding $g_{\mathrm{cw}}$ and gas concentrations at leaf surfaces.

$$
g_{\mathrm{t} w-\mathrm{vCF}}=\frac{E_{\mathrm{T}} \cdot\left(1-\frac{w_{\mathrm{i}}+w_{\mathrm{a}}}{2}\right)}{w_{\mathrm{i}}-w_{\mathrm{a}}}=\frac{E_{\mathrm{T}} \cdot(1-\bar{w})}{\Delta w}
$$

Q8

The main assumptions in equation (1) are that: the total gas exchange occurs through a single pathway between the inside of the leaf and the atmosphere; the ternary correction is applied to the whole flux; and the gradient that drives the exchange is that between the internal $\left(w_{\mathrm{i}}\right)$ and external ambient $\left(w_{\mathrm{a}}\right)$ concentrations. Equation (1) does not separate stomatal, cuticular and boundary layer fluxes for calculating $g_{\mathrm{tw}}$. However, in practice, $E_{\mathrm{T}}$ is the sum of water vapour passing through the stomata $\left(E_{\mathrm{s}}\right)$ and the cuticle $\left(E_{\mathrm{c}}\right)$

$$
E_{\mathrm{T}}=E_{\mathrm{s}}+E_{\mathrm{c}}
$$

and the last is not affected by ternary effects as are the stomata.

In the electrical resistance analogue to leaf gas exchange, the total conductance to $\mathrm{H}_{2} \mathrm{O}$ and $\mathrm{CO}_{2}$ includes stomatal $\left(g_{\text {sw }}\right.$ and $\left.g_{s c}\right)$, cuticular $\left(g_{\mathrm{cw}}\right.$ and $\left.g_{\mathrm{cc}}\right)$ and boundary layer $\left(g_{\mathrm{bw}}\right.$ and $\left.g_{\mathrm{bc}}\right)$ conductances, where $g_{\mathrm{s}}$ and $g_{\mathrm{c}}$ operate in parallel and $g_{\mathrm{b}}$ in series with them (Fig. 1b,c)

$$
g_{\mathrm{tw}}=\frac{1}{\frac{1}{g_{\mathrm{sw}}+g_{\mathrm{cw}}}+\frac{1}{g_{\mathrm{bw}}}} \wedge g_{\mathrm{tc}}=\frac{1}{\frac{1}{g_{\mathrm{sc}}+g_{\mathrm{cc}}}+\frac{1}{g_{\mathrm{bc}}}}
$$

Combined cuticular and stomatal conductances to $\mathrm{H}_{2} \mathrm{O}$ form the total conductance to $\mathrm{H}_{2} \mathrm{O}$ through the surface of the leaf $\left(g_{\mathrm{lw}}\right)$ that is without the boundary layer

$$
g_{\mathrm{lw}}=g_{\mathrm{cw}}+g_{\mathrm{sw}}
$$

In the vCF calculations, $g_{c w}$ is neglected and $g_{\text {sw }}$ approximates to $g_{\mathrm{lw}}$. Taking $g_{\mathrm{tw}}$ from equation (1) and rearranging equation (3)

$$
g_{\mathrm{sw}-\mathrm{vCF}} \approx g_{\mathrm{lw}}=\frac{1}{\frac{1}{g_{\mathrm{tw}}}-\frac{1}{g_{\mathrm{bw}}}}=\frac{g_{\mathrm{tw}} \cdot g_{\mathrm{bw}}}{g_{\mathrm{bw}}-g_{\mathrm{tw}}}
$$

Then, stomatal conductance to $\mathrm{CO}_{2}\left(g_{s c}\right)$ is calculated using the ratio of $\mathrm{H}_{2} \mathrm{O}$ and $\mathrm{CO}_{2}$ diffusivities in air ${ }^{19}$. The ratio $g_{\mathrm{sw}} / g_{\mathrm{sc}}$ is taken as a constant value, estimated to be between 1.58 (ref. ${ }^{20}$ ) and 1.62 , commonly taken as 1.6 (ref. ${ }^{21}$ ). Thus, $g_{s c}=g_{s w} / 1.6$ and then taking $g_{\text {sw-vCF }}$ from equation (5)

$$
g_{\mathrm{sc}-\mathrm{vCF}} \approx \frac{g_{\mathrm{lw}}}{1.6}=\frac{g_{\mathrm{sw}-\mathrm{vCF}}}{1.6}
$$

One of the implications of using the result from equation (5) in this way is that the ratios of cuticular and stomatal conductances to
$\mathrm{H}_{2} \mathrm{O}$ and $\mathrm{CO}_{2}$ are (incorrectly) considered to be the same. However, estimates of $g_{\mathrm{cw}} / g_{\mathrm{cc}}$ are one order of magnitude greater than $g_{\mathrm{sw}} / g_{\mathrm{sc}}$, varying in a range between 20 and 40 (ref. ${ }^{14}$ ). Thus, while the assumption of negligible cuticular conductance to $\mathrm{H}_{2} \mathrm{O}$ has been a practical one, it can lead to significant overestimates of $\mathrm{CO}_{2}$ conductance and subsequent parameters.

The composition of, and transport pathways through, the cuticle (epidermis, cell wall, waxes and so on) that generate these differences are non-trivial ${ }^{22-24}$ and are not treated here. The cuticle and its components are treated as a single resistance as is common in gas exchange modelling.

The cuticular conductance to $\mathrm{CO}_{2}\left(g_{c c}\right)$, is conventionally assumed to be zero. As $g_{c c}$ is an order of magnitude lower than $g_{\mathrm{cw}}$ (ref. ${ }^{14}$ ), this typically only results in a minor error in the calculations. Then, $g_{\mathrm{tc}-\mathrm{vCF}}$ is calculated taking $g_{\mathrm{sc}}$ from equation (6) and rearranging equation (3)

$$
g_{\mathrm{tc}-\mathrm{vCF}} \approx \frac{1}{\frac{1}{g_{\mathrm{sc}}+g_{\mathrm{ccc}}}+\frac{1}{g_{\mathrm{bc}}}}=\frac{g_{\mathrm{bc}} \cdot g_{\mathrm{sc}}}{g_{\mathrm{sc}}+g_{\mathrm{bc}}}
$$

with the main source of error coming from equations (5) and (6).

Direct measurement of $\left[\mathrm{CO}_{2}\right]$ in the air flowing over the leaf allows us to determine the net assimilation rate $\left(A_{\mathrm{T}}\right)$ (ref. $\left.{ }^{2}\right) . \mathrm{CO}_{2}$ concentration inside the leaf $\left(C_{\mathrm{i}}\right)$ is conventionally calculated using the value of $g_{\mathrm{tc}-\mathrm{vCF}}$ obtained from equation (7) and

$$
C_{\mathrm{i}-\mathrm{vCF}}=\frac{\left(g_{\mathrm{tc}}-\frac{E}{2}\right) \cdot C_{\mathrm{a}}-A_{\mathrm{T}}}{g_{\mathrm{tc}}+\frac{E}{2}}
$$

Equation (8) takes into account the ternary effect ${ }^{1}$ (vCF) of the collisions between $\mathrm{H}_{2} \mathrm{O}$ and $\mathrm{CO}_{2}$ molecules when they are passing through stomata in opposing flows. However, the term $E$ in equation (8) should refer only to the portion $E_{\mathrm{s}}$ of the total evaporation which is crossing through stomatal pores. Equation (8) has the same assumptions as discussed for equation (1).

Also, it is important to consider that leaf gas exchange occurs through the adaxial and abaxial leaf faces. The outgoing gases are typically mixed before analysis (Fig. 1a) and the results used to calculate the gas exchange parameters according to the vCF equations (henceforth Gas-Mix vCF). Gas-Mix vCF calculations have the same assumptions discussed above plus the forced presumption of a single $C_{\mathrm{i}}$ in the leaf and single values for other gas exchange parameters.

\section{Deriving more precise theory}

Looking at leaf gas exchange more precisely (Fig. 1b,c), $E_{\mathrm{T}}$ is the water flux from the leaf surface, at vapour concentration $\left(w_{\mathrm{s}}\right)$, crossing the boundary layer to the atmosphere $\left(w_{\mathrm{a}}\right)$. Then, taking into account the ternary effect on the boundary layer transect the following expression is obtained for the humidity at the leaf surface (derived in Supplementary Note 2)

$$
w_{\mathrm{s}}=\frac{\frac{E_{\mathrm{T}}}{g_{\mathrm{bw}}} \cdot\left(1-\frac{w_{\mathrm{a}}}{2}\right)+w_{\mathrm{a}}}{1+\frac{E_{\mathrm{T}}}{2 \cdot g_{\mathrm{bw}}}}
$$

The total outward flux of $\mathrm{H}_{2} \mathrm{O}$ vapour at the surface of the leaf comes from the stomata and the cuticle. Then, adapting equation (3) for the total conductance to $\mathrm{H}_{2} \mathrm{O}\left(g_{\mathrm{tw}}\right)$ by including the ternary effects and cuticular transpiration and conductance (Supplementary Note 3 ), the total leaf conductance to water vapour is given by

$$
g_{\mathrm{tw}}=\frac{1}{\left[\frac{E_{\mathrm{c}}}{w_{\mathrm{i}}-w_{\mathrm{s}}}\right]+\left[\frac{E_{\mathrm{s}}-E_{\mathrm{s}} \cdot \bar{w}_{\mathrm{s}}}{w_{\mathrm{i}}-w_{\mathrm{s}}}\right]}+\frac{1}{\left[\frac{E_{\mathrm{T}}-E_{\mathrm{T}} \cdot \bar{w}_{\mathrm{w}}}{w_{\mathrm{s}}-w_{\mathrm{a}}}\right]}=\frac{1}{\frac{w_{\mathrm{i}}-w_{\mathrm{s}}}{E_{\mathrm{T}}-E_{\mathrm{s}} \cdot\left(\frac{w_{i}+w_{\mathrm{s}}}{2}\right)}+\frac{1}{g_{\mathrm{bw}}}}
$$

Equation (10) for $g_{\text {tw }}$ is identical to equation (3) with the conductances defined to include their associated ternary effects (see 


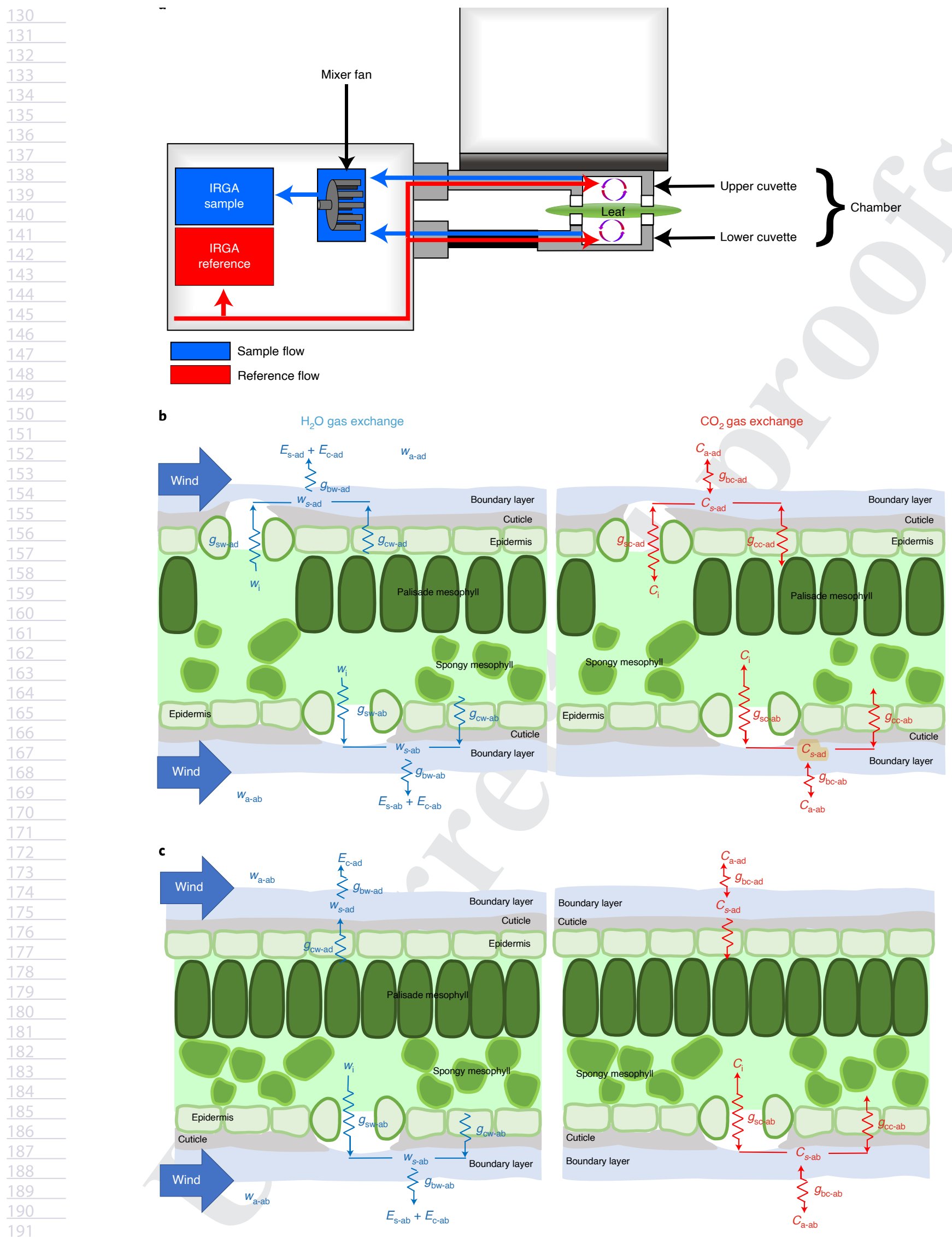

Q9 Fig. 1 | a-c, Typical gas exchange analyser setup (a) and gas exchange diagram from an amphistomatous leaf (b) and a hypostomatous leaf (c). IRGA, Q10 infrared gas analysis. 
Supplementary Notes 3 and 2) but differs from the vCF theory equation (1), which is an approximation.

Analogous to $g_{\text {tw }}$ we have total conductance to $\mathrm{CO}_{2}$, which includes ternary effects, cuticular $\mathrm{CO}_{2}$ flux $\left(A_{\mathrm{c}}\right)$ and stomatal $\mathrm{CO}_{2}$ flux $\left(A_{\mathrm{s}}\right)$

$$
g_{\mathrm{tc}}=\frac{1}{\left[\frac{A_{\mathrm{c}}}{C_{\mathrm{s}}-C_{\mathrm{i}}}\right]+\left[\frac{A_{\mathrm{s}}+E_{\mathrm{s}} \cdot \bar{c}_{\mathrm{s}}}{C_{\mathrm{s}}-C_{\mathrm{i}}}\right]}+\frac{1}{\left[\frac{A_{\mathrm{T}}-E_{T} \cdot \overline{\mathrm{c}}_{\mathrm{a}}}{C_{\mathrm{d}}-C_{\mathrm{s}}}\right]}
$$

Equation (11) is identical to equation (3) for total conductance to $\mathrm{CO}_{2}\left(g_{\mathrm{tc}}\right)$ (see Supplementary Notes 4 and 5).

Then, the $\mathrm{CO}_{2}$ concentration at the leaf surface $\left(C_{\mathrm{s}}\right)$ is (Supplementary Note 4)

$$
C_{s}=\frac{g_{\mathrm{bc}} \cdot C_{\mathrm{a}}-A_{\mathrm{T}}-\frac{E_{\mathrm{T}}}{2} \cdot C_{\mathrm{a}}}{g_{\mathrm{bc}}+\frac{E_{\mathrm{T}}}{2}}
$$

This introduces the equation for calculating $C_{\mathrm{i}}$, taking into account cuticular conductance to $\mathrm{CO}_{2}$ and ternary corrections through the boundary layer and stomatal pathways (Supplementary Note 5)

$$
C_{\mathrm{i}}=\frac{C_{s} \cdot\left(g_{\mathrm{sc}}+g_{\mathrm{cc}}-\frac{E_{\mathrm{s}}}{2}\right)-A_{\mathrm{T}}}{g_{\mathrm{sc}}+g_{\mathrm{cc}}+\frac{E_{\mathrm{s}}}{2}}
$$

By analogy with the vCF theory, Ball ${ }^{25}$ obtained similar equations for $C_{\mathrm{s}}$ and $C_{\mathrm{i}}$ but did not differentiate between $E_{\mathrm{T}}$ and $E_{\mathrm{s}}$ nor $g_{\mathrm{sc}}$ and $g_{c c}$; and $g_{s c}$ in Ball's derivation is based on the calculations from vCF theory equations (5) and (6).

Arranging equations (9)-(13) with parameters in the form usually measured in gas exchange devices, we obtain (derivation in Supplementary Note 6)

$$
C_{\mathrm{i}}=C_{\mathrm{s}}-\frac{A_{\mathrm{T}}+C_{\mathrm{s}} \cdot E_{\mathrm{T}}-C_{\mathrm{s}} \cdot D \cdot g_{\mathrm{cw}}}{G_{\mathrm{i}}-\alpha \cdot g_{\mathrm{cw}}}
$$

where

$$
\begin{aligned}
& D=w_{\mathrm{i}}-w_{\mathrm{s}} \\
& G_{\mathrm{i}}=\frac{E_{\mathrm{T}}}{1.6 \cdot\left(w_{\mathrm{i}}-w_{\mathrm{s}}\right)} \cdot\left(1-\bar{w}_{\mathrm{s}}\right)+\frac{E_{\mathrm{T}}}{2} \\
& \bar{w}_{\mathrm{s}}=\frac{w_{\mathrm{i}}+w_{\mathrm{s}}}{2} \\
& w_{\mathrm{s}}=\frac{w_{\mathrm{a}}+\left(1-\frac{w_{\mathrm{a}}}{2}\right) \frac{E_{\mathrm{T}}}{g_{\mathrm{bw}}}}{1+\frac{E_{\mathrm{T}}}{2 \cdot 8_{\mathrm{bw}}}} \\
& \alpha=\frac{1-\bar{w}_{\mathrm{s}}}{1.6}+\frac{D}{2}-\beta \\
& \beta=\frac{g_{\mathrm{cc}}}{g_{\mathrm{cw}}}
\end{aligned}
$$

Then, expanding the term $E_{\mathrm{s}}$ in equation (10)

$$
g_{\mathrm{tw}}=\frac{1}{\frac{w_{\mathrm{i}}-w_{\mathrm{s}}}{E_{\mathrm{T}}-\left[E_{\mathrm{T}}-g_{\mathrm{cw}-\mathrm{T}} \cdot\left(w_{\mathrm{i}}-w_{\mathrm{s}}\right)\right] \cdot\left(\frac{w_{\mathrm{i}}+w_{\mathrm{s}}}{2}\right)}+\frac{1}{g_{\mathrm{bw}}}}
$$

In equations (14) and (15) the only unknown values are the cuticular conductances to $\mathrm{H}_{2} \mathrm{O}\left(g_{\mathrm{cw}}\right)$ and $\mathrm{CO}_{2}\left(g_{\mathrm{cc}}\right)$.

Equations (9)-(15) represent a new way of calculating gas exchange parameters, modified structurally from the widely used vCF theory.

\section{Model for calculating $\boldsymbol{g}_{\mathrm{cw}}$}

The omission of $g_{\mathrm{cw}}$ in $g_{\mathrm{tw}-\mathrm{vCF}}$ is the major cause of a miscalculation (knowing $g_{\mathrm{cw}} \neq 0$ ) in the conventional $C_{\mathrm{i}-\mathrm{VCF}}$ calculation. This error in the vCF theory has been used to calculate a correction factor taken as $g_{\mathrm{cw}}$ (refs. ${ }^{11,12}$ ). However, this approach to calculating $g_{\mathrm{cw}}$ carries the assumptions made in the vCF theory and assumes that the sole mismatch is from neglecting $g_{\mathrm{cw}}$. Our proposed theory does not involve such assumptions and includes $g_{\mathrm{cw}}$ from the outset of the derivation rather than as a correction. If $C_{\mathrm{i}}$ and $\beta$ are known, equation (14) can be rearranged to solve for $g_{\mathrm{cw}}$ directly

$$
g_{\mathrm{cw}}=\frac{\left(C_{\mathrm{s}}-C_{\mathrm{i}}\right) \cdot G_{\mathrm{i}}-\left(A_{\mathrm{T}}+C_{\mathrm{s}} \cdot E_{\mathrm{T}}\right)}{\left(C_{\mathrm{s}}-C_{\mathrm{i}}\right) \cdot \alpha-C_{\mathrm{s}} \cdot D}
$$

However, in most cases $C_{\mathrm{i}}$ is unknown. Here, we propose a model developed from equation (14) that allows us to estimate $g_{\mathrm{cw}}$ when $C_{\mathrm{i}}$ is unknown, using adaxial and abaxial leaf gas exchange measurements.

Adding physiological parameters representing the ratio of $\mathrm{CO}_{2}$ concentrations at the adaxial and abaxial evaporative sites by $\kappa=C_{\mathrm{i}-}$ ${ }_{\mathrm{ad}} / C_{\mathrm{i}-\mathrm{ab}}$ and the ratio of $g_{\mathrm{cw}}$ on the abaxial and adaxial surface by $\gamma=g_{\text {cw-ab }} / g_{\text {cw-ad }}$, we obtain

$$
\begin{aligned}
& C_{s-\mathrm{ad}}-\frac{A_{\mathrm{T}-\mathrm{ad}}+C_{\mathrm{s}-\mathrm{ad}} \cdot E_{\mathrm{T}-\mathrm{ad}}-C_{s-\mathrm{ad}} \cdot D_{\mathrm{ad}} \cdot g_{\mathrm{cw}-\mathrm{ab}} \cdot \gamma}{G_{\mathrm{i}-\mathrm{ad}}-\alpha_{\mathrm{ad}} \cdot g_{\mathrm{cw}-\mathrm{ab}} \cdot \gamma} \\
& =\left(C_{s-\mathrm{ab}}-\frac{A_{\mathrm{T}-\mathrm{ab}}+C_{\mathrm{s}-\mathrm{ab}} \cdot E_{\mathrm{T}-\mathrm{ab}}-C_{\mathrm{s}-\mathrm{ab}} \cdot D_{\mathrm{ab}} \cdot g_{\mathrm{cw}-\mathrm{ab}}}{G_{\mathrm{i}-\mathrm{ab}}-\alpha_{\mathrm{ab}} \cdot g_{\mathrm{cw}-\mathrm{ab}}}\right) \cdot \kappa
\end{aligned}
$$

where the subscripts ad and ab denote adaxial and abaxial surfaces of the leaf. Equation (17) is then solved for $g_{c w}$ (full derivation in Supplementary Note 7)

$$
a \cdot g_{\mathrm{cw}-\mathrm{ab}}^{2}+b \cdot g_{\mathrm{cw}-\mathrm{ab}}+c=0
$$

where

$$
\begin{aligned}
& a=\left(C_{\mathrm{s}-\mathrm{ad}}-C_{\mathrm{s}-\mathrm{ab}} \cdot \kappa\right) \cdot \alpha_{\mathrm{ad}} \cdot \alpha_{\mathrm{ab}} \cdot \gamma+\kappa \cdot C_{\mathrm{s}-\mathrm{ab}} \cdot D_{\mathrm{ab}} \cdot \alpha_{\mathrm{ad}} \cdot \gamma \\
& -C_{\mathrm{s}-\mathrm{ad}} \cdot D_{\mathrm{ad}} \cdot \alpha_{\mathrm{ab}} \cdot \gamma \\
& b=\left(C_{\mathrm{s}-\mathrm{ad}} \cdot D_{\mathrm{ad}} \cdot G_{\mathrm{i}-\mathrm{ab}} \cdot \gamma+\left(A_{\mathrm{T}-\mathrm{ad}}+C_{\mathrm{s}-\mathrm{ad}} \cdot E_{\mathrm{T}-\mathrm{ad}}\right) \cdot \alpha_{\mathrm{ab}}\right) \\
& -\left(C_{\mathrm{s}-\mathrm{ad}}-C_{s-\mathrm{ab}} \cdot \kappa\right) \cdot\left(\alpha_{\mathrm{ad}} \cdot G_{\mathrm{i}-\mathrm{ab}} \cdot \gamma+G_{\mathrm{i}-\mathrm{ad}} \cdot \alpha_{\mathrm{ab}}\right) \\
& -\kappa \cdot\left(C_{\mathrm{s}-\mathrm{ab}} \cdot D_{\mathrm{ab}} \cdot G_{\mathrm{i}-\mathrm{ad}}+\left(A_{\mathrm{T}-\mathrm{ab}}+C_{\mathrm{s}-\mathrm{ab}} \cdot E_{\mathrm{T}-\mathrm{ab}}\right) \cdot \alpha_{\mathrm{ad}} \cdot \gamma\right) \\
& c=\left(C_{s-\mathrm{ad}}-C_{\mathrm{s}-\mathrm{ab}} \cdot \kappa\right) \cdot G_{\mathrm{i}-\mathrm{ad}} \cdot G_{\mathrm{i}-\mathrm{ab}}-\left(A_{\mathrm{T}-\mathrm{ad}}+C_{s-\mathrm{ad}} \cdot E_{\mathrm{T}-\mathrm{ad}}\right) \\
& \cdot G_{\mathrm{i}-\mathrm{ab}}+\kappa \cdot\left(A_{\mathrm{T}-\mathrm{ab}}+C_{\mathrm{s}-\mathrm{ab}} \cdot E_{\mathrm{T}-\mathrm{ab}}\right) \cdot G_{\mathrm{i}-\mathrm{ad}}
\end{aligned}
$$

and the lesser positive root is taken.

Our model to calculate $g_{c w}$ is based on using the uneven impact of $g_{\mathrm{cw}}$ and $g_{\mathrm{cc}}$ on total conductance to $\mathrm{H}_{2} \mathrm{O}\left(g_{\mathrm{tw}}\right)$ and $\mathrm{CO}_{2}\left(g_{\mathrm{tc}}\right)$ on each surface of the leaf to find $g_{\mathrm{cw}}$ for one surface. The ratio of abaxial and adaxial $g_{\mathrm{cw}}(\gamma)$ distributes the influence of $g_{\mathrm{cw}}$ on each surface. However, the actual value of $\gamma$ is unknown, so for simplicity a value of one is assumed. The rationale for this assumption is discussed and tested later in the paper.

A requirement for using equation (18) is that the experimental setup must provide conditions that allow inference of the value of $\kappa$. Most gas exchange measurements assume that $C_{\mathrm{i}-\mathrm{ad}}=C_{\mathrm{i}-\mathrm{ab}}$, that is $\kappa=1$, and for usual gas exchange measurements, the assumption is adequate $^{26-30}$. However, $\kappa$ can vary depending on the environmental conditions and leaf characteristics ${ }^{31,32}$.

A compilation of $\kappa$ values reported for different species under different conditions is shown in Supplementary Note 8 . The highest value of $\kappa$ found was $1.18\left(\left|\Delta C_{\mathrm{i}}\right| \approx 11 \mathrm{ppm}\right)$, when a Helianthus annuus leaf was exposed to $<100 \mathrm{ppm}$ of ambient $\left[\mathrm{CO}_{2}\right]$; and the lowest $\kappa$ value found was $0.88\left(\left|\Delta C_{\mathrm{i}}\right| \approx 25 \mathrm{ppm}\right)$, when a Eucalyptus pauciflora leaf was exposed to $3 \mathrm{~h}$ of photo-inhibition. However, under non-stressed light conditions and at $\mathrm{CO}_{2}$ concentrations near that of the atmosphere, $\kappa$ values were on average $1.00 \pm 0.05$. For simplicity, then, our experimental setup was established in conditions and ranges of $C_{\mathrm{a}}$ where $\kappa$ can be expected to be close to one and we use $\kappa=1$ in our calculations. To test the impact of $\kappa$ in the calculations, $\kappa$ was varied within reasonable bounds (Extended Data Fig. 1 ). The results of this evaluation are discussed later in the paper.

\section{Cuticular conductance to $\mathrm{H}_{2} \mathrm{O}$}

Adaxial and abaxial gas exchange was measured independently on Capsicum annuum leaves and equation (18) used with $\kappa$ set to unity, 


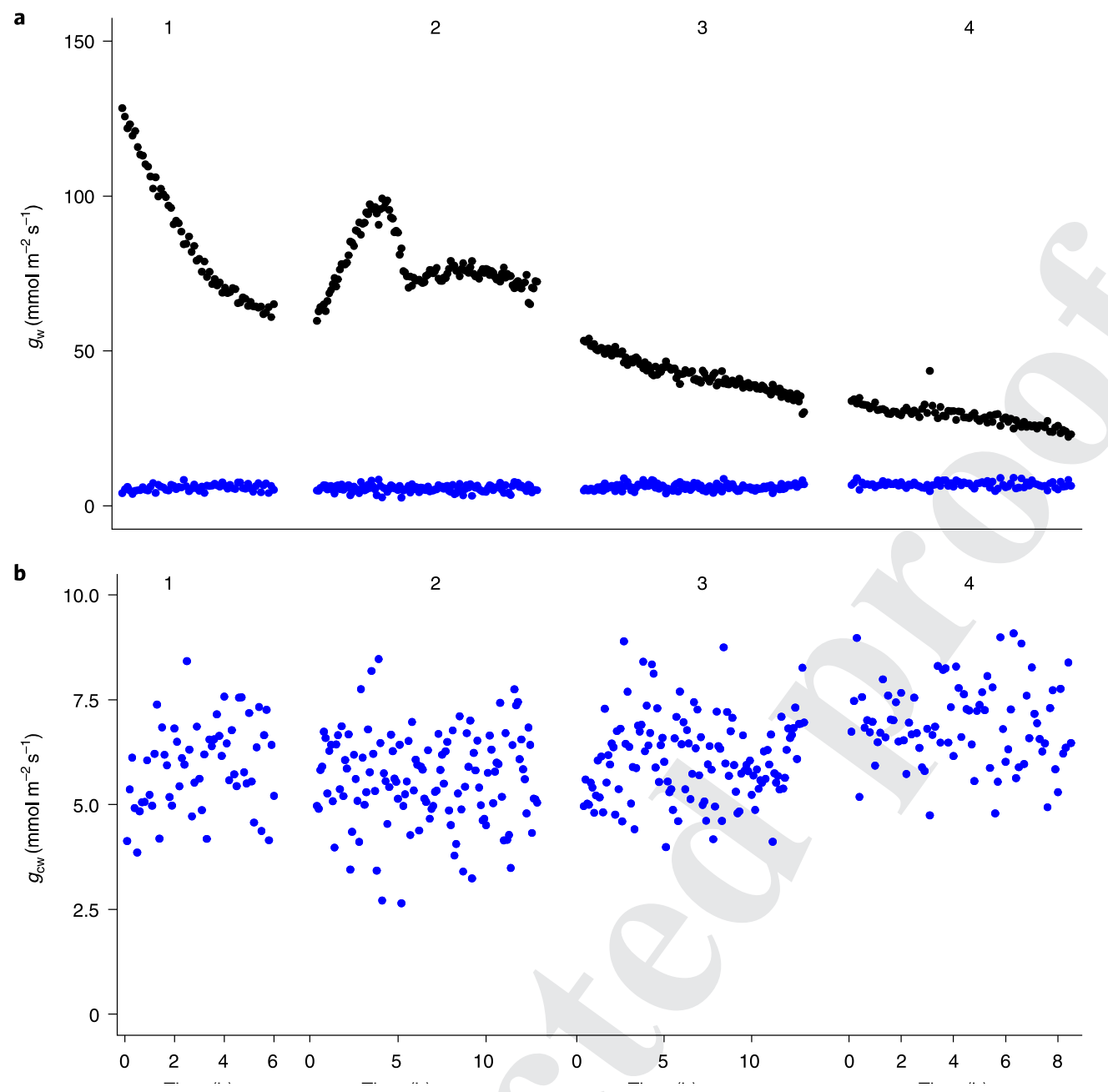

Fig. 2 | Cuticular conductance calculated for single leaf surfaces for four leaves of the same $C$. annuum plant. Four different leaves from the same $C$. annuum plant (1, 2, 3 and 4), with measurements of $6,13,13$ and $10 \mathrm{~h}$ under the same light, $\left[\mathrm{CO}_{2}\right]$ and air saturation deficit $\left(n_{(1,2,3,4)}=60,126,120\right.$ and 85). a, Total leaf surface conductance $\left(g_{\mathrm{lw}-\mathrm{T}}=g_{\mathrm{lw}-\mathrm{ad}}+g_{\mathrm{lw}-\mathrm{ad}}\right.$, black dots) and calculated cuticular conductance for a single surface with $\kappa=1$ (blue dots). $\mathbf{b}$, Close-up of the calculated cuticular conductance. Each point is a single measurement repeated every 6 min. The dataset of each leaf was divided into sections of $1 \mathrm{~h}$ to analyse changes in the calculated $g_{\mathrm{cw}}$. No significant differences were found between time sections within measurements of each leaf; no significant differences were found between the average values of leaves 1, 2 and 3; leaf 4 showed a significantly higher $g_{\mathrm{cw}}$ than the other leaves (statistical analysis: two-sided Student's $t$-test with $P<0.05$; Supplementary Note 9).

to calculate $g_{\mathrm{cw} \cdot}$ Cuticular conductance to water $\left(g_{\mathrm{cw}}\right)$ of each leaf was found to be constant regardless of changes in evaporation rate and natural stomatal aperture changes (circadian rhythm) during the day (Fig. 2; Methods). These observations are consistent with reports by Hoad et al. ${ }^{33}$ and Boyer ${ }^{11,14}$ that cuticular conductance to $\mathrm{H}_{2} \mathrm{O}$ is stable while the leaf retains turgor. Correction of $g_{\mathrm{sw}}$ was $5-20 \%$ of the total $g_{l_{\mathrm{w}}}\left(g_{\mathrm{lw}-\mathrm{T}}=g_{\mathrm{lw}-\mathrm{ad}}+g_{\mathrm{lw}-\mathrm{ad}}\right)$ for C. annuum plants during these experiments.

In a study comparing species, leaf cuticular conductance to $\mathrm{H}_{2} \mathrm{O}$ was calculated for C. annuum, Ginkgo biloba, Gossypium hirsutum and Nicotiana tabacum plants (Fig. 3).

Our results were compared with a common method for measuring adaxial and abaxial minimum conductance, which involves measuring gas exchange after long dark adaptation with and without ABA on the leaf surface ${ }^{13}$. From lengthy experiments conducted on G. hirsutum and C. annuum, adding ABA and placing the plants in the dark, the minimum conductance $\left(g_{1 \mathrm{w}-\min }+\mathrm{ABA}\right)$ for the adaxial surface of the leaf was practically the same as our calculated $g_{\mathrm{cw}}$ The value of $g_{\mathrm{lw} \text {-min }}+\mathrm{ABA}$ for C. annuum was $5.7 \mathrm{mmol} \mathrm{m}^{-2} \mathrm{~s}^{-1}$ and the $g_{\mathrm{cw}}$ was $5.6 \mathrm{mmolm}^{-2} \mathrm{~s}^{-1}$. For $G$. hirsutum the values were 1.9 and $1.63 \mathrm{mmol} \mathrm{m}^{-2} \mathrm{~s}^{-1}$, respectively.
Our calculated cuticular conductances were always below the adaxial and abaxial minimum conductance (Table 1) as one would expect assuming a tiny stomatal aperture. In three of these species, the conductance of both leaf surfaces was measured after $12 \mathrm{~h}$ of dark adaptation $\left(g_{\mathrm{lw}-\mathrm{min}}\right)$ when stomata had closed. It was found that, in C. annuum leaves, the cuticular conductance comprised $94 \%$ of $g_{\text {lw-min }}\left(g_{c w} / g_{\text {lw-min }}\right)$ on the upper surface and $60 \%$ on the lower surface (in other words, $6 \%$ and $40 \%$ of vapour was coming from almost closed stomata, respectively). The corresponding values of $g_{\mathrm{cw}} / g_{\mathrm{lw}-\mathrm{min}}$ for G. hirsutum were $90 \%$ and $75 \%$, and in G. biloba were $8 \%$ in the lower surface and $100 \%$ for the astomatous surface, the last largely confirming almost closed stomata as the other water source.

Leaves of $C$. annuum plants grown under water-stress conditions (Fig. 3, C. annuum 3-D and 4-D) were also measured after long dark adaptation. The water stress of our C. annuum plants was managed by watering them only when the soil water potential reached $-0.1 \mathrm{MPa}$. It was found that $g_{\mathrm{Iw} \text {-min }}$ for these plants, measured when fully rehydrated, was two to three times less than in well-watered plants and the stomatal component of the water loss was higher. Compared with minimum conductance measured in the dark, the cuticular conductance was $50 \%$ of the abaxial surface conductance and $75 \%$ 


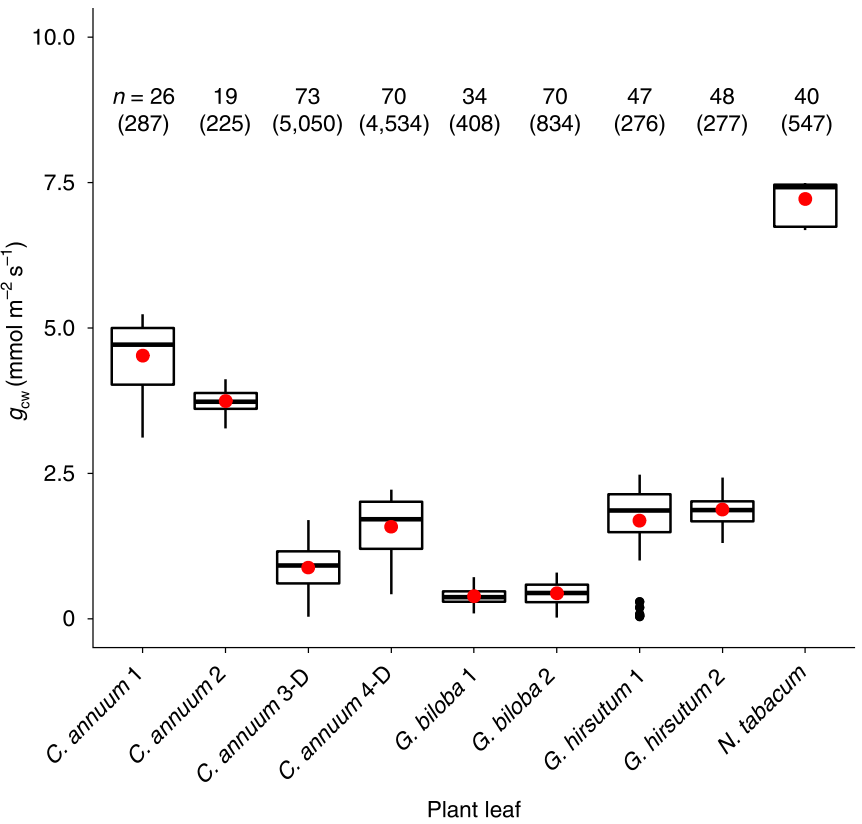

Fig. 3 | Average cuticular conductance calculated for a single leaf surface for leaves of different plants $(\kappa=1)$. C. annuum 3-D and 4-D were grown in drought conditions. Red dots are the mean values of the dataset, boxes represent the range of data between the second and third quartiles of frequency $(I \mathrm{Q}=\mathrm{Q} 3-\mathrm{Q} 2)$, the horizontal lines in the boxes represent the median of the dataset, vertical lines represent the range of maximum and minimum values excluding outliers and black dots are outliers

213 (outliers are: $<\mathrm{Q} 2-1.5^{\star} \mathrm{IQ}$ or $>\mathrm{Q} 3+1.5^{\star} \mathrm{IQ}$ ). Each observation $(n)$ used for the calculations is the average of 12 to 72 measurements under stable conditions for a period of $6 \mathrm{~min}$. The total number of observations is shown in parentheses, each leaf being measured for 2-7 $\mathrm{h}$.

for the adaxial surfaces, a lower percentage than in well-watered plants. Other researchers have reported similar results with plants grown in well-watered and drought-stressed conditions ${ }^{34-37}$.

\section{Effect of $C_{\mathrm{a}}$, temperature and humidity on $\boldsymbol{g}_{\mathrm{cw}}$ calculation}

It was found that simultaneous changes in $C_{\mathrm{a}}$ at both surfaces had no significant effect on the $g_{\mathrm{cw}}$ calculation, when plants were in stable gas exchange conditions (Extended Data Fig. 2). Neither temperature nor atmospheric saturation deficit affected our calculations of cuticular conductance to $\mathrm{H}_{2} \mathrm{O}$ on well-watered plants (Extended Data Fig. 2).

A stable $g_{\mathrm{cw}}$ of about $5 \pm 0.5$ (mean \pm s.d.) $\mathrm{mmol} \mathrm{m}^{-2} \mathrm{~s}^{-1}$ for one surface of the leaf $(\gamma=1)$ was found for a $C$. annuum leaf regardless of changes in air temperature. Our measurements were carried out at leaf temperatures in the range $20-29^{\circ} \mathrm{C}$ and are consistent with the minor changes in cuticle permeability reported for isolated cuticles within this range ${ }^{38}$.

There was no significant change in the calculated $g_{\mathrm{cw}}$ in a large range of ambient relative humidity (Extended Data Fig. 2) although other studies on isolated cuticles ${ }^{39}$ have reported important variations in the cuticular permeability resulting from changes in relative humidity. These variations seem to be species dependent, with permeabilities at $100 \%$ relative humidity between 1.4 and ten times higher than at $0 \%$ relative humidity. There is still little information about the mechanism of this variation and the effect that isolation of the cuticular waxes has on the measurements.

\section{Cuticular conductance to $\mathrm{CO}_{2}$}

On an astomatous surface, it should be possible to measure the actual $g_{c c}$; however, the conductance to $\mathrm{CO}_{2}$ through the cuticle is usually so low that the signal is too weak and noise is overwhelming. The measurements of assimilation rate through the astomatous surfaces in G. biloba leaves were at the limit of the instrumental noise, averaging $0.015 \pm 0.018$ (mean \pm s.d.) $\mu \mathrm{mol} \mathrm{m}^{-2} \mathrm{~s}^{-1}$. This makes it challenging to estimate a reliable ratio of $g_{\mathrm{cc}} / g_{\mathrm{cw}}=\beta$ directly.

The literature suggests values for $\beta$ of between 0.025 and 0.05 , depending on the species and growth conditions ${ }^{6,11,14}$. The average positive assimilation rate occurring through the cuticle $\left(A_{\mathrm{c}}\right)$ in $G$. biloba leaves means that in our calculations a residual $g_{\text {sw }}$ is assigned to the astomatous surfaces if $\beta$ is set as 0 . This residual $g_{\text {sw }}$ is due to the calculations where $\mathrm{CO}_{2}$ must pass through one of the two pathways and $\beta=0$ mathematically blocks the cuticular pathway. If we estimate a $\beta$ value that satisfies the condition of obtaining an average $g_{\mathrm{sw}}$ for the adaxial surface equal to 0 , we obtain $\beta \approx 0.05$. Even though the $\beta$ value found is within the range suggested in the literature, the measurements of $A_{c}$ were not precise enough for us to validate our $\beta$ value.

An additional issue with the calculation explained above is that it was made assuming $\gamma=1$ and technically we could make the same calculation assigning any reasonable $\beta>0$ and correcting the $g_{\text {sw }}$ by varying $\gamma$. Further in the paper, $\gamma$ is estimated as 1.06 for G. biloba leaves (adaxial surface with lower conductance) using measurements of minimum conductance in the dark. Using $\gamma=1.06$ and recalculating $\beta$ to make adaxial $g_{\text {sw }}=0$, we obtain an insignificantly higher $\beta$ that still approximates to $\beta \approx 0.05$ but again with insufficient precision of measurement of $A_{c}$.

Correcting our calculations using $\beta=0.05$ in $G$. biloba leaves has no significant influence on $g_{\mathrm{cw}}$ estimation, on $C_{\mathrm{i}}$ or on any other gas exchange parameter calculation during positive net assimilation rate. Nor does the value of $\beta$ impact the calculations made in the other species used in our experiments. However, knowing that $g_{c c} \neq 0, \beta$ values within the range reported in the literature are appropriate in the absence of a known $\beta$.

\section{Elucidating the model}

To calculate $g_{\mathrm{cw}}$ using our model (equation (18)), the values of $A_{\mathrm{T}}, E_{\mathrm{T}}$ plus $g_{\mathrm{lw}}$ and $g_{\mathrm{lc}}$ for the adaxial and abaxial surface are balanced using a mediated $\kappa$ value. Thus, to calculate $g_{\mathrm{cw}}$ the mathematics require a difference between leaf surfaces in at least one of these parameters, as usually happens. However, this also implies that the calculation relies on the precision of $\left[\mathrm{H}_{2} \mathrm{O}\right]$ and $\left[\mathrm{CO}_{2}\right]$ measurements from the upper and lower cuvettes and any failure in the precision between cuvette measurements results in a miscalculation of $g_{\mathrm{cw}^{*}}$ Our experiments showed that the main possible source of error in our model and method is the drift in the equipment used to calculate $\mathrm{H}_{2} \mathrm{O}$ and $\mathrm{CO}_{2}$ balances (Supplementary Note 10).

During our experiments the calculated $g_{\mathrm{cw}}$ was stable with a reliable value whenever the experimental setup allowed inference of $\kappa$. The impact of $\kappa$ in the calculation using equation (17) depends on the magnitude of $g_{\mathrm{lc}}$ on each surface. In the case of a hypostomatous leaf, the astomatous surface has a very low $\mathrm{CO}_{2}$ conductance in comparison with the surfaces with stomata. Conventionally, internal $\left[\mathrm{CO}_{2}\right]$ is defined as the $\left[\mathrm{CO}_{2}\right]$ calculated in the evaporative site ${ }^{31}$ but, in the astomatous surface, internal $\left[\mathrm{CO}_{2}\right]$ and $\mathrm{C}_{\mathrm{s}}$ are physically separated. This physical separation offers great resistance to $\mathrm{CO}_{2}$ diffusion, creating the conditions where the value of $\kappa$ has a small impact on our calculations.

It is possible to evaluate the latter concept from our measurements made in hypostomatous G. biloba leaves. Our first approach was to estimate $g_{\mathrm{cw}}$ assuming $\kappa=1$. Later, we imposed $\kappa \approx 0.9$ equivalent to the $\Delta C_{\mathrm{i}}$ found by Parkhurst et al. ${ }^{29}$ when only one surface of an amphistomatous leaf was fed with $\mathrm{CO}_{2}$. The change did not affect the calculated value of $g_{\mathrm{cw}}$. We evaluated $\kappa$ in a range from 0.9 to 1.1 and found insignificant variation in the calculation of $g_{\mathrm{cw}}$ in $G$. biloba leaves (Extended Data Fig. 1). In contrast, the same analysis in an amphistomatous leaf of N. tabacum showed a big impact in the same range. 
Table 1 | Comparison of measured minimum conductance to $\mathrm{H}_{2} \mathrm{O}$, calculated $g_{\mathrm{cw}}$ using equation (18), the dark- $g_{\mathrm{sw}}$ calculated as $g_{\mathrm{lw}-\mathrm{min}}-\boldsymbol{g}_{\mathrm{cw}}$, Dark- $g_{\mathrm{sw}}$ ratio and stomatal density ratio

\begin{tabular}{|c|c|c|c|c|c|c|}
\hline Plant & Surface & $g_{\mid w-\min }$ & $g_{\mathrm{cw}}$ & Dark- $g_{s w}$ & Dark- $g_{\mathrm{sw}}$ & Stomatal density \\
\hline & & $\left(\mathrm{mmol} \mathrm{m} \mathrm{m}^{-2} \mathrm{~s}^{-1}\right)$ & $\left(\mathrm{mmol} \mathrm{m} \mathrm{m}^{-2} \mathrm{~s}^{-1}\right)$ & $\left(\mathrm{mmolm} \mathrm{m}^{-2} \mathrm{~s}^{-1}\right)$ & $(\%)$ & $(\%)$ \\
\hline \multirow[t]{2}{*}{ C. annuum } & Adaxial & 8.5 & 5.6 & 2.9 & 26 & 10 \\
\hline & Abaxial & 13.8 & 5.6 & 8.2 & 74 & 90 \\
\hline \multirow[t]{2}{*}{ G. biloba $a^{a}$} & Adaxial & 0.5 & 0.35 & 0.15 & 6 & 0 \\
\hline & Abaxial & 2.75 & 0.35 & 2.4 & 94 & 100 \\
\hline \multirow[t]{2}{*}{ G. biloba ${ }^{b}$} & Adaxial & 0.5 & 0.49 & 0.01 & 0 & 0 \\
\hline & Abaxial & 2.75 & 0.49 & 2.26 & 100 & 100 \\
\hline \multirow[t]{2}{*}{ G. hirsutum } & Adaxial & 2.5 & 1.63 & 0.88 & 17 & 25 \\
\hline & Abaxial & 6 & 1.63 & 4.38 & 83 & 75 \\
\hline
\end{tabular}

${ }^{a}$ Calculation assuming $\beta=0 .{ }^{b}$ Calculated using $\beta=0.05$.
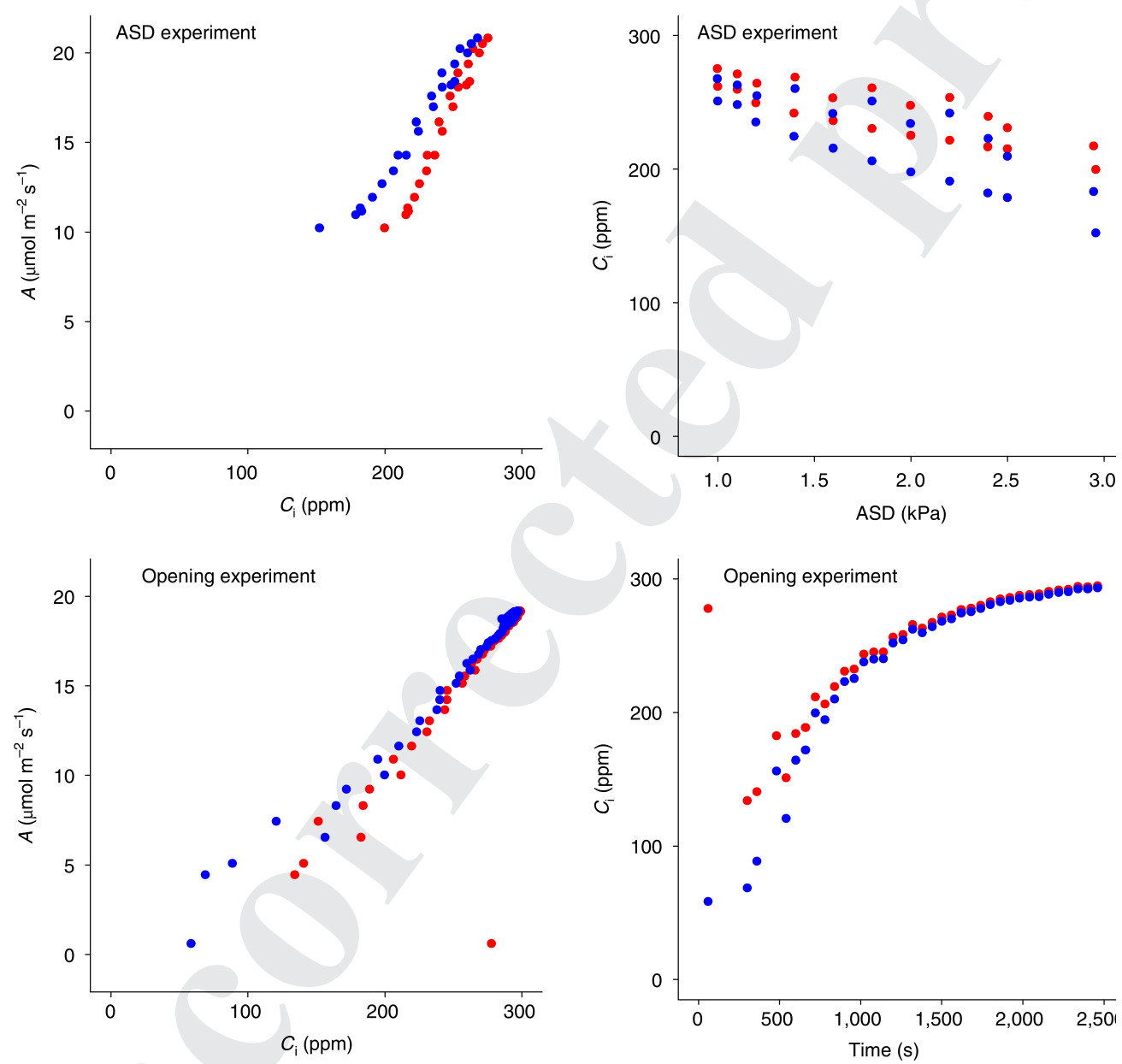

Fig. 4 | Gas exchange parameters calculated using equations (9)-(15) and the vCF theory for experiments changing ASD and stomatal aperture from dark to light (opening) on C. annuum plants. Red dots show the results of using the Gas-Mix vCF calculation and the blue dots the calculations using equations (9)-(15) accounting for cuticular gas fluxes. Each point in the ASD experiment is a single measurement taken under stable leaf gas exchange conditions. Each point in the opening experiment is a single measurement taken every $1 \mathrm{~min}$; initial measurements are more spaced due to the rapid change of gas exchange conditions and subsequent IRGA matches for pairing the sample and reference IRGA.

In a general analysis, the value of the parameter $\kappa$ affects the calculated relationship between $g_{1 \mathrm{w}}, g_{\mathrm{sw}}$ and $g_{\mathrm{cw}}$; the ternary correction on the $\mathrm{CO}_{2}$ fluxes; and the $\mathrm{CO}_{2}$ gradients between the stomatal cavity and the leaf surface. In the calculations made in this study, we assume that due to our experimental setup the $\left[\mathrm{CO}_{2}\right]$ at the sites of evaporation is the same for both sides of a leaf $(\kappa=1)$. This is likely to be the case when there is negligible resistance to $\mathrm{CO}_{2}$ diffusion inside the leaf and the ambient conditions are equal in both surfaces but not necessarily restricted to that condition (Supplementary Note 8 ). On the other hand, gradients across a leaf can be of the order of $20 \mathrm{ppm}$ or more if $A_{\mathrm{T}}$ at one surface is constrained to zero ${ }^{29}$. Additionally, the assumption of $\kappa=1$ may not hold when the plant 

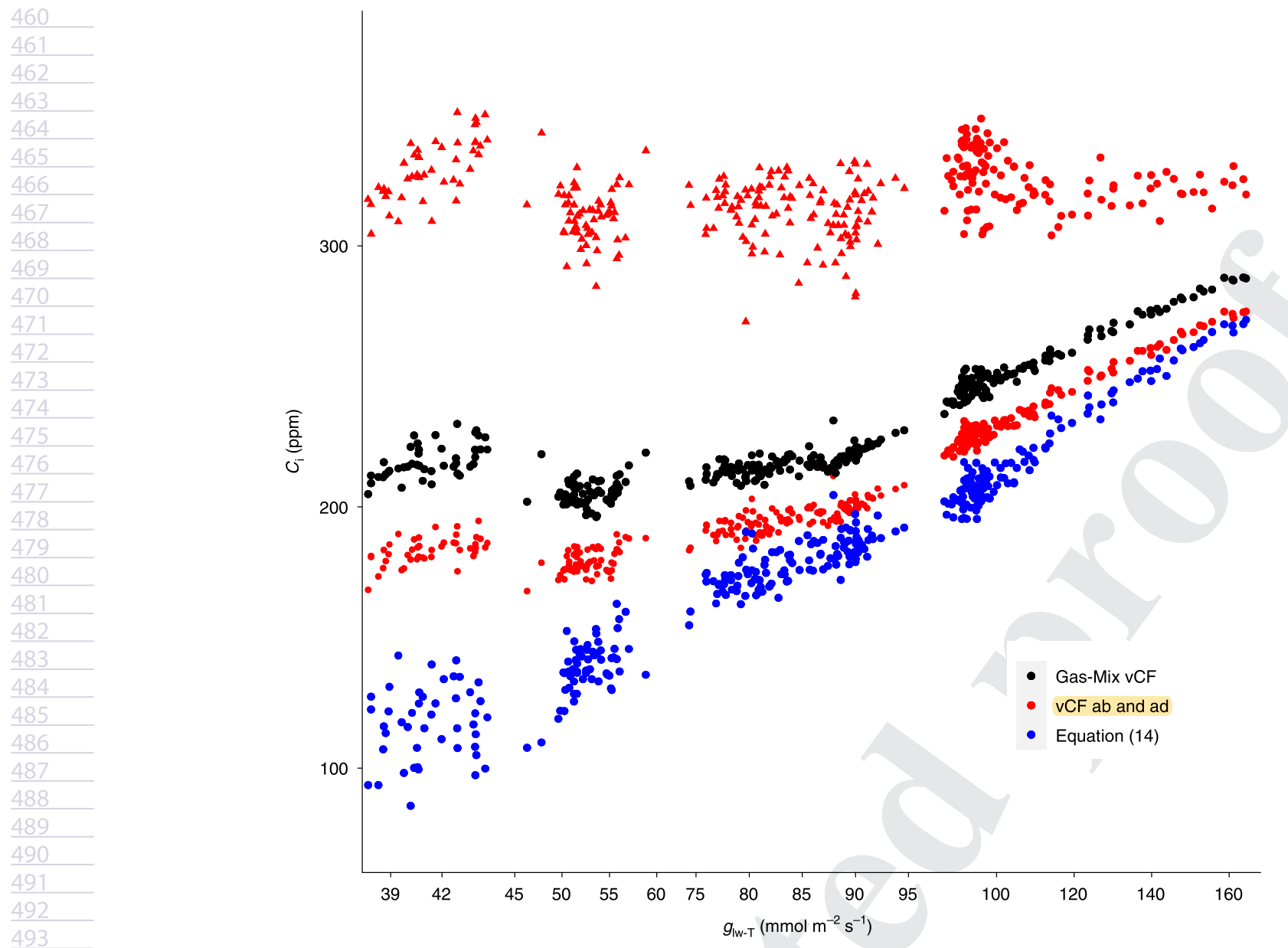

Fig. 5 | Comparison of $C_{i}$ calculation using vCF, Gas-Mix vCF and equation (14) calculations. $\mathrm{CO}_{2}$ concentration inside a $C$. annuum leaf using equation (8) (VCF) for the adaxial (red triangles) and abaxial (red circles) faces of a leaf and the Gas-Mix vCF calculation using a mixture of the gases from both faces of the leaf (black circles). Blue circles are the calculation of $\mathrm{C}_{i}$ using equation (14) with $\kappa=1$. The total conductance to $\mathrm{H}_{2} \mathrm{O}$ through both surfaces of the leaf $\left(g_{\mid \mathrm{W}-\mathrm{T}}\right)$ is displayed as the $x$ axis: sections are different periods of the day; each section has a different scale in the $x$ axis to make them readable. One point is a single measurement, taken every 6 min. Further analysis of Fig. 5 in Supplementary Note 11.

species has thick leaves or if there is an internal or external physical impediment to free diffusion in the leaf ${ }^{26,27}$. For instance, see the contrast between Xanthium strumarium and Zea mays reported by Farquhar and Raschke ${ }^{32}$. In our experimental setup, we used a combination of plants and environmental conditions where it is reasonable to assume that $\kappa \approx 1$ and we tested the possible error arising from this assumption (Extended Data Fig. 1).

Another aspect that might affect the calculations is the difference between the adaxial and abaxial $g_{\mathrm{cw}}$. Although the two leaf surfaces might have different cuticular structures, direct values of cuticular conductance from the astomatous surfaces have been used as an approximation of the cuticular conductance of the opposite surface $^{5,6}$. We took this initial approach assigning $\gamma=1$ for equation (18) because the actual difference is unknown. However, a recent study ${ }^{40}$ found that the adaxial and abaxial cuticles of a hypostomatous leaf have different evaporative rates under similar environmental conditions. This might indicate significant differences in $g_{\mathrm{cw}}$ for the adaxial and abaxial surfaces.

To address this, we used dark measurements to obtain the $g_{\mathrm{lw}-\mathrm{min}}$ of the adaxial and abaxial surfaces. Then, the calculated $g_{\mathrm{cw}}$ and $g_{\text {lw-min }}$ were used to isolate the dark- $g_{\text {sw }}$ from both surfaces. Ratios of stomatal conductance in the dark were compared with the stomatal density ratios and used to test whether differences in $g_{\mathrm{lw} \text {-min }}$ between the abaxial and adaxial surfaces were explained by stomatal densities (Table 1). It was found that a clear majority of the differences in the adaxial and abaxial $g_{1 \mathrm{w}-\min }$ were explained by stomatal density imbalances.

The difference between the stomatal density ratio and the $g_{\text {Iw-min }}$ ratio might be explained by uneven stomatal closure on the two surfaces, differences in adaxial and abaxial $g_{\mathrm{cw}}$ or a mixture of these two factors. To evaluate the impact of differences in adaxial and abaxial $g_{\mathrm{cw}}$, we considered the extreme case where the whole difference between these ratios is due to uneven $g_{\mathrm{cw}}$ in the adaxial and abaxial surfaces. We found values of $\gamma\left(g_{\mathrm{cw}-\mathrm{ab}} / g_{\mathrm{cw}-\mathrm{ad}}\right)$ of 1.18 , 1.09 and 1.06 for C. annuum, G. hirsutum and G. biloba, respectively. Using equation (18) and these $\gamma$ values to recalculate the total $g_{\mathrm{cw}}\left(g_{c w-T}=g_{c w-a d}+g_{c w-a b}\right)$, it was found that $g_{\mathrm{cw}-\mathrm{T}}$ of our plants was increased by $12.5 \%$ in C. annuum, $5 \%$ in G. hirsutum and $3 \%$ in $G$. biloba. The other gas exchange parameters were not significantly affected when corrected by $\gamma$. An analysis of the impact of $\gamma$ values of between 0.7 and 1.4 is presented in (Extended Data Fig. 3).

In the model presented in equation (18) we included the ratio of abaxial to adaxial $g_{\text {cw }}(\gamma)$, which is usually unknown. This introduces a level of uncertainty to the calculations of total cuticular conductance to water $\left(g_{c w-T}=g_{c w-a d}+g_{c w-a b}\right)$; however, within reasonable range, $\gamma$ has a minor effect in the calculation of other gas exchange parameters. Our theory gives a tool to evaluate the differences between adaxial and abaxial $g_{c w}$ and the future direction of our research will focus on it. 


\section{Theory insights}

The gas exchange mathematical theory derived in equations (9)-(15) incorporates two new variables in the calculation of gas exchange: $g_{\mathrm{cw}}$ and $g_{\mathrm{cc}}$. The variable $g_{\mathrm{cc}}$ can be expressed in terms of $g_{\mathrm{cw}}$ using the parameter $\beta$ as presented in equation (14) and discussed in the section Cuticular conductance to $\mathrm{CO}_{2}$.

The theory can be applied to any gas exchange measurement, independently of whether the intention is to estimate $g_{\mathrm{cw}}$ or not. It calculates gas exchange parameters from normal gas exchange measurements provided $g_{\mathrm{cw}}$ is known. For instance, using the default setup of a LI-6800, we performed experiments involving air saturation deficit (ASD) and stomatal opening in response to light using C. annuum leaves where the $g_{\mathrm{cw}}$ was determined in advance. Then, we calculated all the gas exchange parameters using equations (9)(15) (Fig. 4).

In the examples shown in Fig. 4 , the $A-C_{\mathrm{i}}$ relation obtained using equations (9)-(15) is lower than that calculated with the widely used Gas-Mix vCF calculation. These results are consistent with the corrections made by Tominaga et al..$^{15}$ using direct measurements to estimate $C_{\mathrm{i}}$. Calculations made using equations (9)-(15) show more consistency in the $A-C_{\mathrm{i}}$ trend, especially when stomata are not fully open. Similar results were found in experiments conducted in a gas exchange system able to measure adaxial and abaxial surfaces independently (Fig. 5). The vCF equations overestimate $C_{\mathrm{i}}$, which results in in miscalculation of other parameters calculated from it such as mesophyll conductance. Overestimation of $C_{\mathrm{i}}$ under ambient $\left[\mathrm{CO}_{2}\right]$ $\left(C_{\mathrm{a}} \approx 400 \mu \mathrm{mol} \mathrm{mol}^{-1}\right)$ causes underestimation of the mesophyll conductance, for example in calculations based on isotope discrimination techniques ${ }^{41}$ such as those by Tazoe et al. ${ }^{42}$ or Busch et al. ${ }^{43}$.

The proposed physiological model to estimate cuticular conductance (equation (18)) is valuable when the actual $C_{\mathrm{i}}$ is unknown and reasonable assumptions can be made about the relationship between adaxial and abaxial $C_{\mathrm{i}}$ values $(\kappa)$. The application of the model requires a gas exchange system able to measure adaxial and abaxial surfaces independently such as those used by us, Mott and $\mathrm{O}^{\prime}$ Leary ${ }^{27}$, Sharkey et al. ${ }^{44}$, Wong et al. ${ }^{17}$, Parkhurst et al. ${ }^{29}$ and Farquhar and Raschke ${ }^{32}$.

Another example of the use of our theory to calculate $g_{\mathrm{cw}}$ is where $C_{\mathrm{i}}$ is known and then substituted in equation (16). For instance, $C_{\mathrm{i}}$ can be estimated in the way proposed by Sharkey et al. ${ }^{44}$ or with the method used by Tominaga and Kawamitsu ${ }^{12}$ to estimate $g_{\mathrm{cw}}$ but using equation (16) instead. Then all the gas exchange parameters can be calculated as before, using equations (9)-(15).

\section{Conclusion}

The theory developed here adds a more detailed characterization of leaf gas exchange considering the two pathways of transport from inside the leaf to the atmosphere. This innovation adds more detail to gas exchange calculations, including the interaction of cuticular fluxes with the other gas exchange parameters and the effect of ternary corrections in the boundary layer. Also, it was shown that calculations allow a more precise estimation of gas exchange parameters in normal gas exchange measurements without any special setup.

Additionally, we accurately estimated the cuticular conductance to $\mathrm{H}_{2} \mathrm{O}$ for different species using the model derived from our theory. It was shown that atmospheric conditions did not affect our results of cuticular conductance. Differing stomatal densities on adaxial and abaxial surfaces facilitate the use of our instrumentation and experimental setup to estimate cuticular conductance to $\mathrm{H}_{2} \mathrm{O}$ vapour.

\section{Methods}

Gas exchange devices. Two LI-6800 gas exchange analysers (LI-COR) were used simultaneously to evaluate the gas exchange from the adaxial and abaxial surfaces of the leaf. The upper and lower cuvettes of a fluorescence system head of an
LI-6800 were separated; leaving only the upper cuvette connected to the gas analyser system (Extended Data Fig. 4, LI-6800 no. 1). The lower cuvette was connected to the other LI-6800 system (Extended Data Fig. 4, LI-6800 no. 2): its reference and sample gas were isolated after the fan mixer. The connection was made using an adaptor created inhouse (Extended Data Fig. 4) by P. Groeneveld. In addition, experiments were carried out on a gas exchange analysis system (system 1) built by S. C. Wong, which uses two LI-6251 gas analysers (LI-COR) to measure the $\left[\mathrm{H}_{2} \mathrm{O}\right]$ and $\left[\mathrm{CO}_{2}\right]$ of the upper and lower cuvettes independently. System 1 is constantly measuring a reference gas of known concentrations obtaining the actual absolute values from each LI-6251 on each measurement. During all the experiments the pressures of both cuvettes were kept equal, using the pressure and flow control included in the LI-6800s and the independent flow control in system 1.

Plant material. Five species were used in our experiments: C. annuum, $N$. tabacum, H. annuus, G. hirsutum and G. biloba. All the plants, except G. biloba, were kept in a greenhouse under natural light conditions with a temperature of $28^{\circ} \mathrm{C}$ during the day and $20^{\circ} \mathrm{C}$ at night. G. biloba plants were kept in a shadehouse. They were watered twice a day, except those plants under water-stress treatments (Data collection). C. annuum plants were germinated in a germination chamber and transplanted to their final pots after the third true leaf was fully expanded. All the other species were grown in their final pots inside the greenhouse.

We took advantage of two characteristics of C. annuum: (1) C. annuum has a significant difference in the stomatal distributions between the adaxial and abaxial surfaces of the leaf; in our plants, the distribution was about $10 \%$ adaxial, $90 \%$ abaxial. (2) C. annuum plants also possess a strong circadian rhythm in their stomatal behaviour, which was used to measure the effect of different natural variations of stomatal conductance on cuticular conductance calculations under non-stressed conditions.

In addition, experiments to determine the cuticular conductance of other species with different stomatal distributions were performed. The plants were $N$. tabacum, which has a $40 / 60 \%$ distribution of stomatal density on the adaxial and abaxial surface of the leaf; G. hirsutum, which has 25/75\% distribution; G. biloba, which has $0 / 100 \%$; and H. annuus, which has $\sim 50 / 50 \%$ distribution.

Data collection. To evaluate the feasibility of using equation (18) to calculate $g_{\mathrm{cw}}$ two experiments were performed on C. annuum plants using system 1 under light conditions of $1,000 \mu \mathrm{mol} \mathrm{m}^{-2} \mathrm{~s}^{-1}$ : first following the diurnal rhythm over $24 \mathrm{~h}$ at $450 \mathrm{ppm}$ of $\mathrm{CO}_{2}$ and second with $30 \mathrm{~min}$ of measurement under 220, 380, 420 and $880 \mathrm{ppm}$ of $\mathrm{CO}_{2}$. Similar experiments were performed on all the plant species mentioned above using the system with two LI-6800s following the diurnal rhythm over $24 \mathrm{~h}$, setting atmospheric $\left[\mathrm{CO}_{2}\right]$ at $400 \mathrm{ppm}$.

Our results were compared with minimum leaf surfaces conductance after $12 \mathrm{~h}$ in the dark with and without $\mathrm{ABA}$ application. The $\mathrm{ABA}$ was prepared at $10^{-4} \mathrm{M}$ in water. The ABA was applied to the adaxial surfaces of the leaf using a spray.

The 24-h experiments were performed to evaluate the effect of air temperature in the range $20-28^{\circ} \mathrm{C}$ and atmospheric ASD of $0.8-1.8 \mathrm{kPa}$ on our calculations. Additionally, experiments with cycles of temperature and relative humidity were performed setting the air temperature at 19,25 and $35^{\circ} \mathrm{C}$; and atmospheric relative humidity at 40 and $80 \%$.

A set of $C$. annuum plants was grown under water deficit, being watered only when the soil matric potential reached $-0.1 \mathrm{MPa}$, as measured with Decagon MPS-6 devices (Decagon Devices). Two MPS-6 were placed at different depths in each plant pot. Plants were grown under this condition from the third true leaf stage until maturity when the measurements were performed. They were then watered to ensure fully turgid conditions during the test.

Another two sets of experiments were conducted on well-watered C. annuum plants using the system of two LI-6800s to evaluate the impact of changes in stomatal conductance on estimates of $g_{\mathrm{cw}}$. The first involved 11 changes in ASD, from 1 to $3 \mathrm{kPa}$, and then decreasing from 3 to $1 \mathrm{kPa}$, waiting until stomatal conditions were stable at each point. The second consisted of changing the irradiance from 0 to $1,000 \mu \mathrm{mol} \mathrm{m}^{-2} \mathrm{~s}^{-1}$, recording the gas exchange parameters every minute.

To analyse how $C_{\mathrm{i}}$ calculation is affected during all these experiments, $C_{\mathrm{i}}$ was also calculated following the typical method in most gas exchange experiments, which involves effectively mixing the gases from both surfaces (cuvettes) and calculating as per the vCF theory ${ }^{1}$.

Additional experiments were made using the normal setup of a LI-6800 (single chambers) on the same plants for which the cuticular conductance to water was previously determined.

The data analysis was performed using the software RStudio v.1.3.959.

Reporting Summary. Further information on research design is available in the Nature Research Reporting Summary linked to this article.

\section{Data availability}

All generated and analysed data from this study are included in the published article, its Supplementary Information and in the GitHub repository (https://github.com/PlantPhysiologist/When_small_fluxes_ matter-calculating-leaf-gas-exchange). Source data are provided with this paper. 
Received: 17 July 2020; Accepted: 22 January 2021;

\section{0.}

\section{References}

1. von Caemmerer, S. \& Farquhar, G. D. Some relationships between the biochemistry of photosynthesis and the gas exchange of leaves. Planta 153, 376-387 (1981)

2. Gaastra, P Photosynthesis of Crop Plants as Influenced by Light, Carbon Dioxide, Temperature, and Stomatal Diffusion Resistance (H. Veenman, 1959).

3. Sheriff, D. W. Epidermal transpiration and stomatal responses to humidity: some hypotheses explored. Plant Cell Environ. 7, 669-677 (1984).

4. Boyer, J. S. \& Kawamitsu, Y. Photosynthesis gas exchange system with internal $\mathrm{CO}_{2}$ directly measured. Environ. Control Biol. 49, 193-207 (2011).

5. Holmgren, P., Jarvis, P. \& Jarvis, M. Resistances to carbon dioxide and water vapour transfer in leaves of different plant species. Physiol. Plant. 18, 557-573 (1965).

6. Boyer, J. S., Wong, S. C. \& Farquhar, G. D. $\mathrm{CO}_{2}$ and water vapor exchange across leaf cuticle (epidermis) at various water potentials. Plant Physiol. 114 185-191 (1997).

7. Choat, B. et al. Triggers of tree mortality under drought. Nature 558, 531-539 (2018).

8. Blackman, C. J. et al. Toward an index of desiccation time to tree mortality under drought. Plant Cell Environ. 39, 2342-2345 (2016).

9. Hanson, D., Stutz, S. S. \& Boyer, J. S. Why small fluxes matter: the case and approaches for improving measurements of photosynthesis and (photo) respiration. J. Exp. Bot. 67, 3027-3039 (2016).

10. Mizokami, Y., Noguchi, K., Kojima, M., Sakakibara, H. \& Terashima, I. Mesophyll conductance decreases in the wild type but not in an ABA-deficient mutant (aba1) of Nicotiana plumbaginifolia under drought conditions. Plant Cell Environ. 38, 388-398 (2015).

11. Boyer, J. S. Impact of cuticle on calculations of the $\mathrm{CO}_{2}$ concentration inside leaves. Planta 242, 1405-1412 (2015).

12. Tominaga, J. \& Kawamitsu, Y. Cuticle affects calculations of internal $\mathrm{CO}_{2}$ in leaves closing their stomata. Plant Cell Physiol. 56, 1900-1908 (2015).

13. Duursma, R. A. et al. On the minimum leaf conductance: its role in models of plant water use, and ecological and environmental control. New Phytol. 221, 693-705 (2018)

14. Boyer, J. S. Turgor and the transport of $\mathrm{CO}_{2}$ and water across the cuticle (epidermis) of leaves. J. Exp. Bot. 66, 2625-2633 (2015).

15. Tominaga, J., Shimada, H. \& Kawamitsu, Y. Direct measurement of intercellular $\mathrm{CO}_{2}$ concentration in a gas-exchange system resolves overestimation using the standard method. J. Exp. Bot. 69, 1981-1991 (2018)

16. Wong, S. C., Cowan, I. R. \& Farquhar, G. D. Leaf conductance in relation to assimilation in Eucalyptus pauciflora Sieb. ex Spreng: influence of irradiance and partial pressure of carbon dioxide. Plant Physiol. 62, 670-674 (1978).

17. Wong, S. C., Cowan, I. R. \& Farquhar, G. D. Leaf conductance in relation to rate of $\mathrm{CO}_{2}$ assimilation I. Influence of nitrogen nutrition, phosphorus nutrition, photon flux density, and ambient partial pressure of $\mathrm{CO}_{2}$ during ontogeny. Plant Physiol. 78, 821-825 (1985).

18. Farquhar, G. D. \& Sharkey, T. D. Stomatal conductance and photosynthesis. Annu. Rev. Plant Physiol. 33, 317-345 (1982).

19. Moss, D. N. \& Rawlins, S. L. Concentration of carbon dioxide inside leaves. Nature 197, 1320-1321 (1963).

20. Massman, W. J. A review of the molecular diffusivities of $\mathrm{H}_{2} \mathrm{O}, \mathrm{CO}_{2}, \mathrm{CH}_{4}$, $\mathrm{CO}, \mathrm{O}_{3}, \mathrm{SO}_{2}, \mathrm{NH}_{3}, \mathrm{~N}_{2} \mathrm{O}, \mathrm{NO}$, and $\mathrm{NO}_{2}$ in air, $\mathrm{O}_{2}$ and $\mathrm{N}_{2}$ near STP. Atmos. Environ. 32, 1111-1127 (1998).

21. Cowan, I. R. Mass and heat transfer in laminar boundary layers with particular reference to assimilation and transpiration in leaves. Agric. Meteorol. 10, 311-329 (1972).

22. Tredenick, E. C., Farrell, T.W. \& Forster, W. A. Mathematical modelling of hydrophilic ionic fertiliser diffusion in plant cuticles: lipophilic surfactant effects. Plants 8, 202 (2019).

23. Tredenick, E. C., Farrell, T. W. \& Forster, W. A. Mathematical modeling of diffusion of a hydrophilic Ionic fertilizer in plant cuticles: surfactant and hygroscopic effects. Front. Plant Sci. 9, 1888 (2018).

24. Tredenick, E. C., Farrell, T. W., Forster, W. A. \& Psaltis, S. T. P. Nonlinear porous diffusion modeling of hydrophilic ionic agrochemicals in astomatous plant cuticle aqueous pores: a mechanistic approach. Front. Plant Sci. 8, 746 (2017).

25. Ball, J. T. in Stomatal Function (eds Cowan, I. R. et al.) 445-476 (Stanford Univ. Press, 1987).

26. Syvertsen, J. P., Lloyd, J., McConchie, C., Kriedemann, P. E. \& Farquhar, G. D. On the relationship between leaf anatomy and $\mathrm{CO}_{2}$ diffusion through the mesophyll of hypostomatous leaves. Plant Cell Environ. 18, 149-157 (1995).

27. Mott, K. A. \& O'Leary, J. W. Stomatal behavior and $\mathrm{CO}_{2}$ exchange characteristics in amphistomatous leaves. Plant Physiol. 74, 47-51 (1984).
28. Wong, S. C., Cowan, I. R. \& Farquhar, G. D. Leaf conductance in relation to rate of $\mathrm{CO}_{2}$ assimilation II. Effects of short-term exposures to different photon flux densities assimilation. Plant Physiol. 78, 826-829 (1985).

29. Parkhurst, D. F., Wong, S. C., Farquhar, G. D. \& Cowan, I. R. Gradients of intercellular $\mathrm{CO}_{2}$ levels across the leaf mesophyll. Plant Physiol. 86, 1032-1037 (1988).

30. Wong, S. C., Cowan, I. R. \& Farquhar, G. D. Leaf conductance in relation to rate of $\mathrm{CO}_{2}$ assimilation III. Influences of water stress and photoinhibition Plant Physiol. 78, 830-834 (1985).

31. Parkhurst, D. F. Diffusion of $\mathrm{CO}_{2}$ and other gases inside leaves. New Phytol. 126, 449-479 (1994).

32. Farquhar, G. D. \& Raschke, K. On the resistance to transpiration of the sites of evaporation within the leaf. Plant Physiol. 61, 1000-1005 (1978).

33. Hoad, S. P., Grace, J. \& Jeffree, C. E. A leaf disc method for measuring cuticular conductance. J. Exp. Bot. 47, 431-437 (1996).

34. Bakker, J. C. Leaf conductance of four glasshouse vegetable crops as affected by air humidity. Agric. Meteorol. 55, 23-36 (1991).

35. Kerstiens, G. Cuticular water permeability and its physiological significance. J. Exp. Bot. 47, 1813-1832 (1996)

36. Cameron, K. D., Teece, M. A. \& Smart, L. B. Increased accumulation of cuticular wax and expression of lipid transfer protein in response to periodic drying events in leaves of tree tobacco. Plant Physiol. 140 176-183 (2006).

37. $\mathrm{Bi}, \mathrm{H}$. et al. The impact of drought on wheat leaf cuticle properties. $B M C$ Plant Biol. 17, 1-13 (2017).

38. Schreiber, L. Effect of temperature on cuticular transpiration of isolated cuticular membranes and leaf discs. J. Exp. Bot. 52, 1893-1900 (2001).

39. Burghardt, M. \& Riederer, M. in Biology of the Plant Cuticle Vol. 23 (eds Riederer, M. \& Muller, C.) 292-311 (2006)

40. Zhang, Y. et al. A proposed method for simultaneous measurement of cuticular transpiration from different leaf surfaces in Camellia sinensis. Front. Plant Sci. 11, 420 (2020).

41. Evans, J., Sharkey, T., Berry, J. \& Farquhar, G. Carbon isotope discrimination measured concurrently with gas exchange to investigate $\mathrm{CO}_{2}$ diffusion in leaves of higher plants. Funct. Plant Biol. 13, 281-292 (1986).

42. Tazoe, Y., von Caemmerer, S., Estavillo, G. M. \& Evans, J. R. Using tunable diode laser spectroscopy to measure carbon isotope discrimination and mesophyll conductance to $\mathrm{CO}_{2}$ diffusion dynamically at different $\mathrm{CO}_{2}$ concentrations. Plant Cell Environ. 34, 580-591 (2011).

43. Busch, F. A., Holloway-Phillips, M., Stuart-Williams, H. \& Farquhar, G. D. Revisiting carbon isotope discrimination in $\mathrm{C} 3$ plants shows respiration rules when photosynthesis is low. Nat. Plants 6, 245-258 (2020).

44. Sharkey, T. D., Imai, K., Farquhar, G. D. \& Cowan, I. R. A direct confirmation of the standard method of estimating intercellular partial pressure of $\mathrm{CO}_{2}$ Plant Physiol. 69, 657-659 (1982).

\section{Acknowledgements}

We thank CONICYT Doctorado, Becas Chile/2015 Folio 72160160 and the Australian Research Council Centre of Excellence for Translational Photosynthesis for funding part of the research. We also thank P. Groeneveld for technical support and building the LI-6800 connector; S. Chin Wong for providing extra minimum conductance data and technical support; and Australian National University Plant Services for taking care of the plant material.

\section{Author contributions}

D.M.A., H.S-W. and G.D.F. conceived the study. D.M.A. undertook the experimental work and data analysis. D.M.A. and G.D.F. carried out the modelling. D.M.A. wrote the manuscript with help from all authors.

\section{Competing interests}

The authors declare no competing interests.

\section{Additional information}

Extended data is available for this paper at https://doi.org/10.1038/s41477-021-00861-w. Supplementary information The online version contains supplementary material available at https://doi.org/10.1038/s41477-021-00861-w.

\section{Correspondence and requests for materials should be addressed to G.D.F.}

Peer review information Nature Plants thanks David Hanson, Thomas Sharkey and the other, anonymous, reviewer(s) for their contribution to the peer review of this work.

Reprints and permissions information is available at www.nature.com/reprints.

Publisher's note Springer Nature remains neutral with regard to jurisdictional claims in published maps and institutional affiliations.

(c) The Author(s), under exclusive licence to Springer Nature Limited 2021 


\section{NATURE PLANTS}

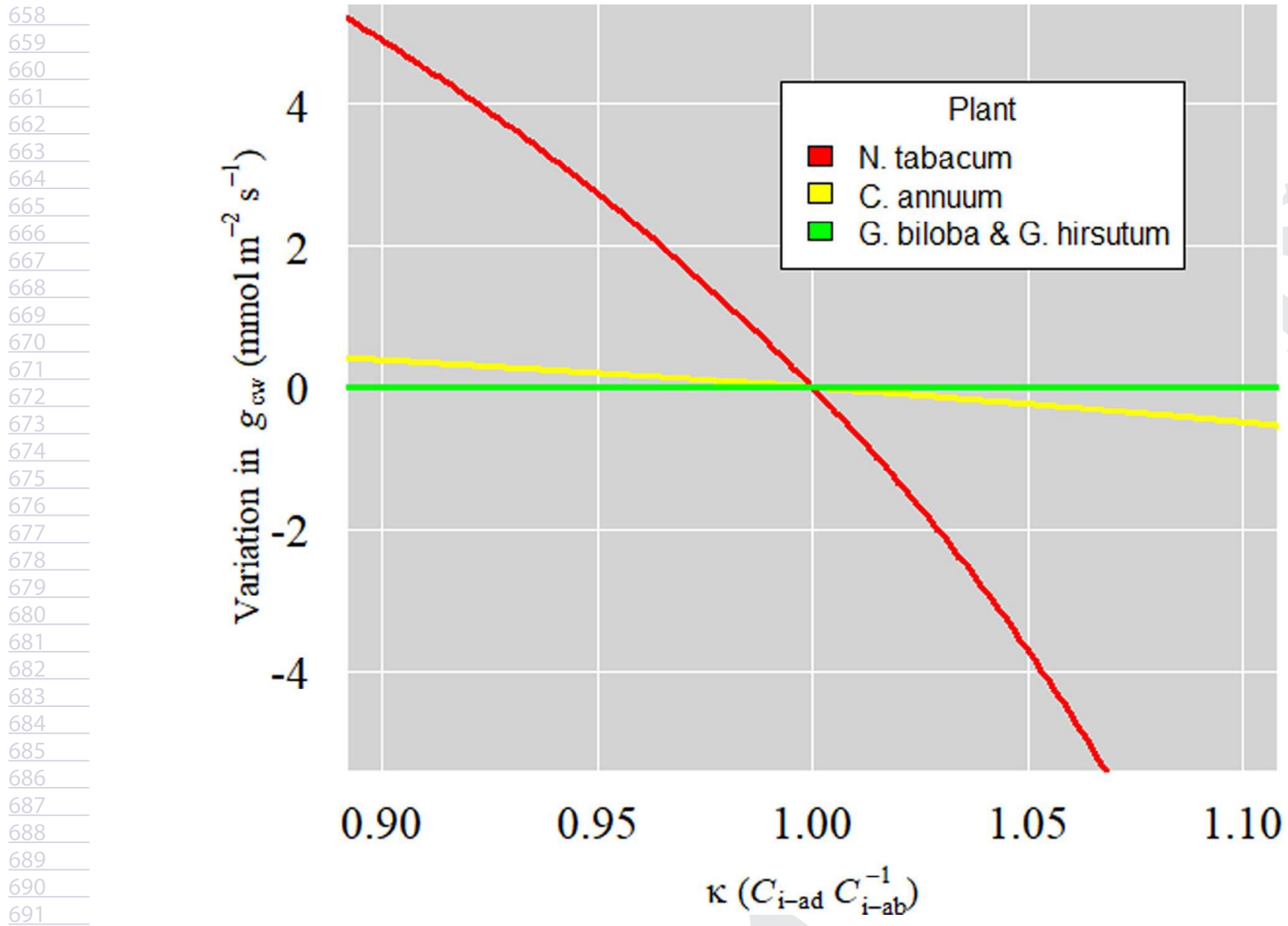

Extended Data Fig. 1 | Effect of $\kappa$ on cuticular conductance calculations. Variation of $g_{\mathrm{cw}}$ calculation if the $\left[\mathrm{CO}_{2}\right]$ at the evaporative sites of the adaxial and abaxial surfaces of the leaf differ (Variation $=g_{\mathrm{cw}}(\kappa)-g_{\mathrm{cw}}(1)$ ). Differences in $\left[\mathrm{CO}_{2}\right]$ in the evaporative site of the adaxial and abaxial surfaces impact gas exchange measurements and calculations to different degrees depending on the external measured values. For the purposes of our study, the impact on the $g_{\mathrm{cw}}$ calculation was tested by keeping measurements of external gas exchange parameters constant and changing $\kappa$. 
a

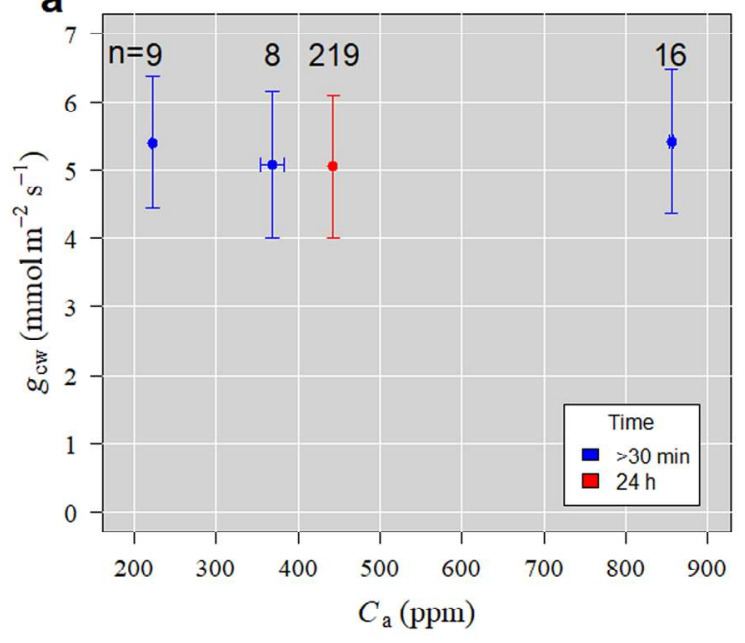

b

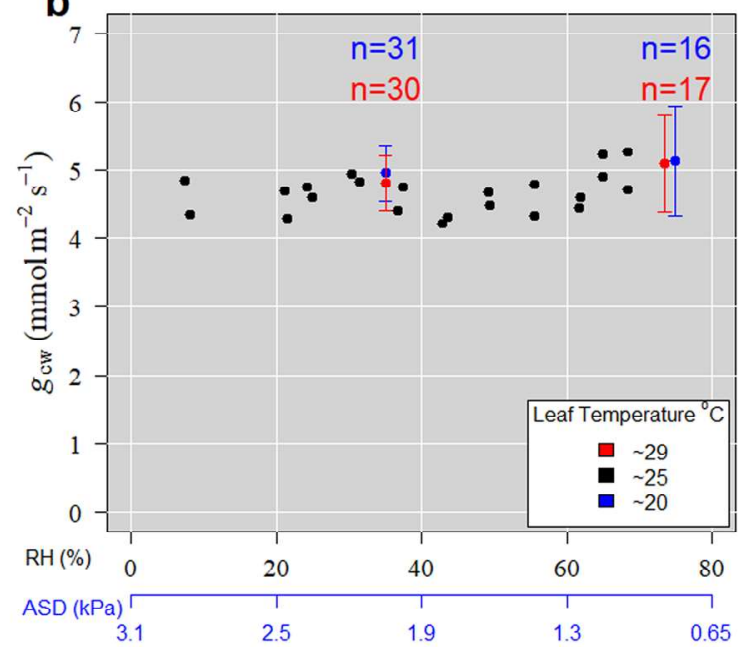

Extended Data Fig. 2 | Average $\boldsymbol{g}_{\mathrm{cw}}$ for a $\boldsymbol{C}$. annuum leaf under different atmospheric conditions (assuming $\boldsymbol{\kappa}=1$ ). a, $g_{\mathrm{cw}}$ measured under different atmospheric $\mathrm{CO}_{2}$ concentrations $\left(\mathrm{C}_{\mathrm{a}}\right)$ over 30 minutes after achieving stable conditions (blue dots) and an average of 24 hours under light at constant $\left[\mathrm{CO}_{2}\right]$ following a diurnal cycle of atmospheric conditions (red dot); and (b) at different temperatures of the leaf and varying relative humidity (RH) also expressed as atmospheric saturation deficits (ASD) for the black dots. In panel (a), each blue point gives the average and standard deviation of 8 to 16 measurements ( $n$ ); the red point gives the average and standard deviation of 239 measurements $(n)$, one measurement being taken every 4 minutes. In panel (b), each red and blue dot gives the average and standard deviation of 16 to 31 measurements ( $n$ ), one measurement taken every 2 minutes; black dots are single measurements under stable gas exchange conditions for at least 25 minutes. The measurements were made from low to high ASD and later from high to low ASD. Graphs (a) and (b) are different leaves. Cuticular conductance $\left(g_{\mathrm{cw}}\right)$ is expressed as the value for one surface and therefore needs to be doubled for the whole leaf $(\gamma=1)$. 

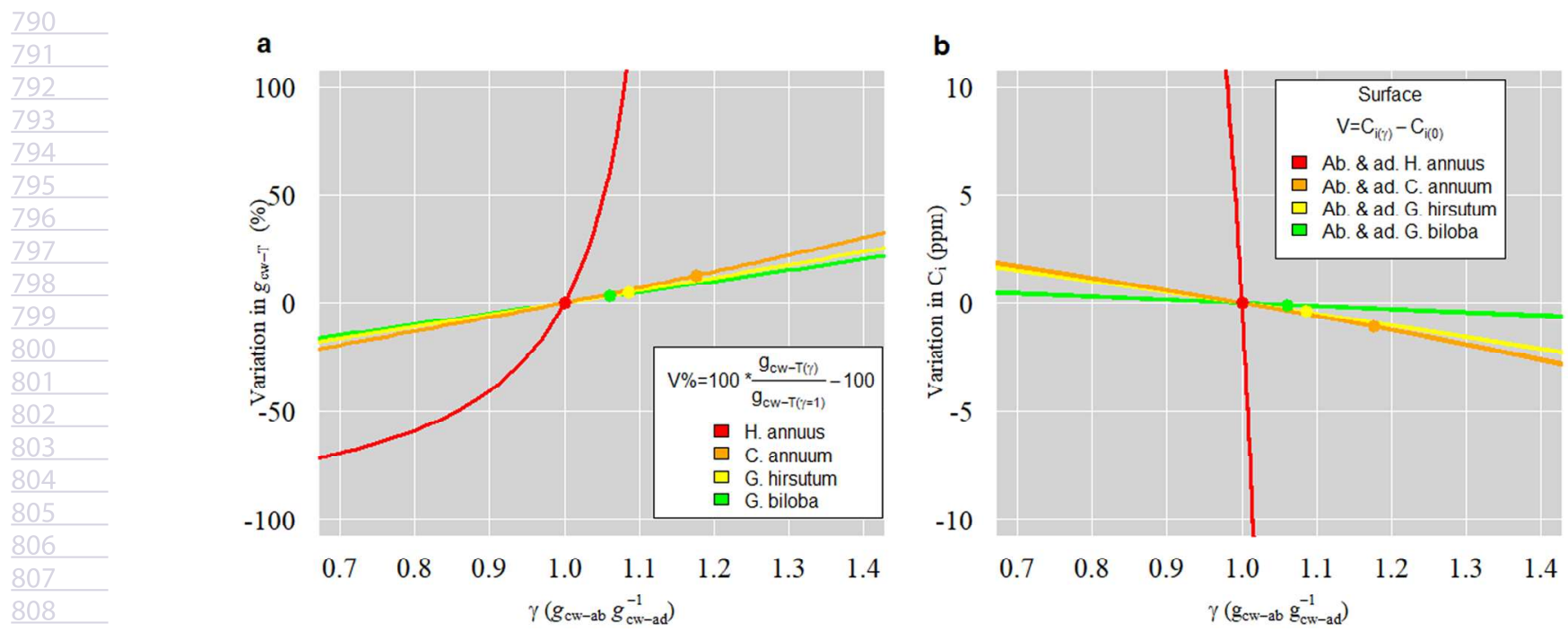

Extended Data Fig. 3 | Effect of possible differences in abaxial and adaxial leaf cuticular conductance. Impact of unequal adaxial and abaxial $g_{\mathrm{cw}}$ in (a) the total cuticular conductance $\left(g_{\mathrm{cw}-\mathrm{T}}=g_{\mathrm{cw}-\mathrm{ad}}+g_{\mathrm{cw}-\mathrm{ab}}\right)$ calculated for a leaf and in (b) the $C_{\mathrm{i}}$ calculation, depending on the proportion of abaxial over adaxial cuticular conductance $(\gamma)$ using as a reference $g_{\mathrm{cw}-\mathrm{T}}$ calculated with $\gamma=1$. A single measurement under stable gas exchange conditions was selected for calculating the variation of each species using $\kappa=1$. The dots show the values using our dark measurements to estimate $\gamma$ for each species. Zhang et al. ${ }^{40}$ measured weight loss via cuticle of detached leaves and they concluded that astomatous surfaces have about $30 \%$ lower evaporation than the cuticle of the surfaces with stomata. It is difficult to extrapolate these evaporation values to $g_{\mathrm{cw}}$ values in growing leaves because the boundary layer conductance and leaf temperature were not provided, and the leaves had already been detached for one hour. Bearing this in mind, we used dark measurements to estimate inequalities between the adaxial and abaxial cuticular conductance, which can be used to quantify a possible $g_{\mathrm{cw}}$ difference between the adaxial and adaxial surfaces. Then, assuming equal stomatal closure on the adaxial and abaxial surface, in $G$. biloba only $6 \%$ of the difference in $g_{\mathrm{lw}}$ was not explained by the stomatal density differences, $8 \%$ for $G$. hirsutum and $15 \%$ for $C$. annuum; the abaxial and adaxial $g_{\text {lw }}$ of $H$. annuus were practically the same in the dark. Using equation (18), it was found that significant variations in $\gamma$ can have a big impact on the calculated total cuticular conductance $\left(g_{\mathrm{cw}-\mathrm{T}}=\right.$ $\left.g_{\mathrm{cw}-\mathrm{dd}}+g_{\mathrm{cw}-\mathrm{ab}}\right)$. The magnitude of this impact depends on how much $\gamma$ affects the $w_{\mathrm{i}}-w_{\mathrm{s}}$ gradient. 


\section{ARTICLES}

NATURE PLANTS

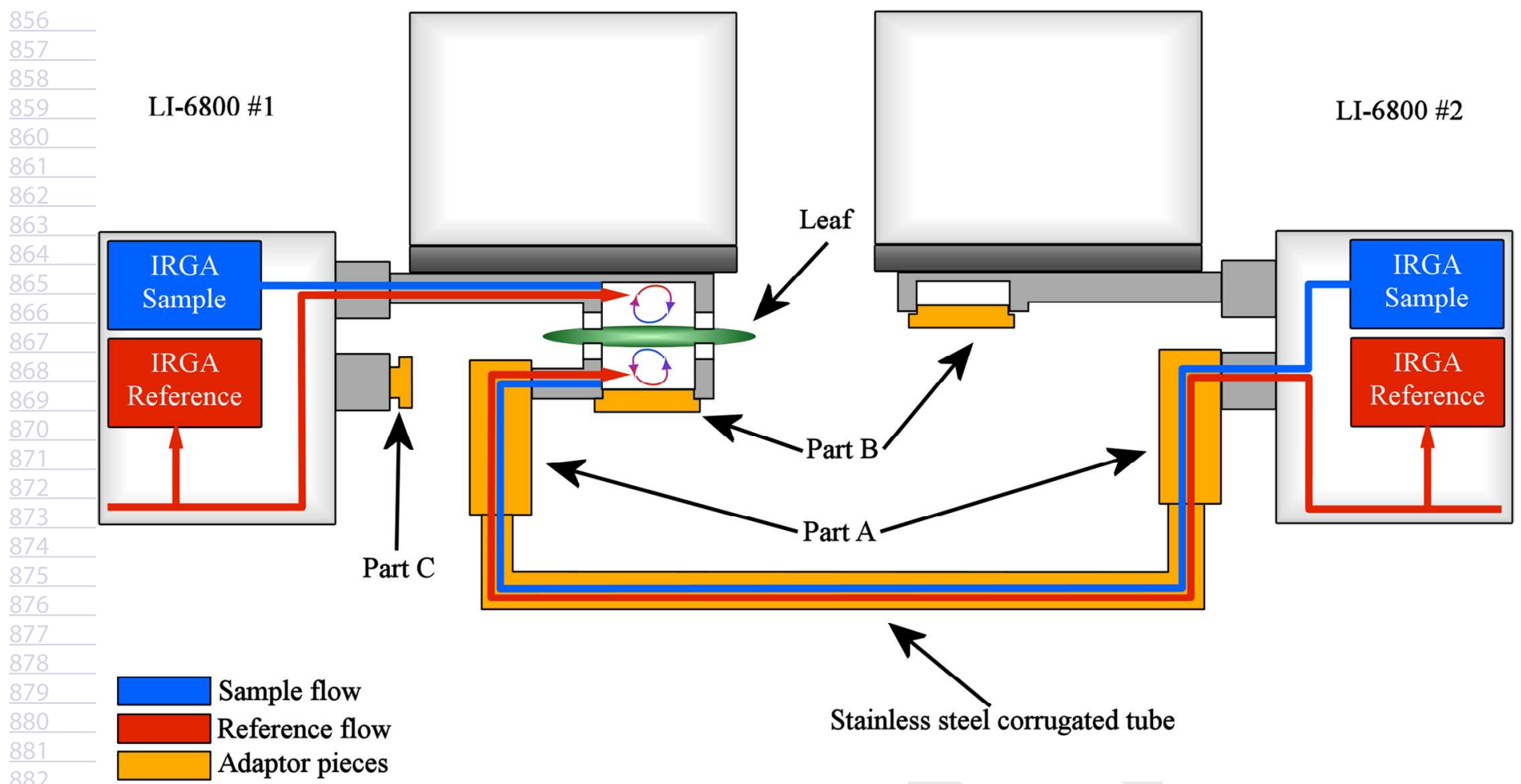

Extended Data Fig. 4 | Diagram of the connection of two LI-6800. Part A: Flanges on the lower cuvette and the second LI-6800. Part B: Lid of the upper cuvette, the lid of the lower cuvette includes two cavities to insert two thermocouples. Part C: Flange to block the flow of gases from and to the lower cuvette. 